\title{
On Structural Patterns of Mechanical Systems with Impacts with One and Two Degrees-of-Freedom
}

\author{
Barbara Blazejczyk-Okolewska \\ Division of Dynamics, Technical University of Lodz, Stefanowskiego 1/15, 90-924 Lodz, Poland \\ Correspondence should be addressed to Barbara Blazejczyk-Okolewska; okolbar@p.lodz.pl
}

Received 20 July 2012; Accepted 5 December 2012

Academic Editor: Jun-Juh Yan

Copyright ( 2013 Barbara Blazejczyk-Okolewska. This is an open access article distributed under the Creative Commons Attribution License, which permits unrestricted use, distribution, and reproduction in any medium, provided the original work is properly cited.

\begin{abstract}
Structural patterns of mechanical systems with impacts with one degree-of-freedom and two degrees-of-freedom, with elastic connections, have been identified and described. For their identification, a general method proposed by the author has been applied. This method uses (i) a matrix representation of the system with impacts, (ii) procedures that enable generations of all combinations of such systems as well as their identification and elimination of redundant equivalent combinations, and (iii) a procedure for elimination of disconnected systems.
\end{abstract}

\section{Introduction}

Systems with one degree-of-freedom belong to the simplest mechanical systems. These systems have been analyzed by numerous authors (e.g., [1-44]), and their dynamics have been investigated in a wide range. The following issues have been considered in the above-mentioned publications: steady periodic motions, subharmonic motions, stability of vibrations, elastic and plastic impacts, time and phase characteristics, behavior of the system under an influence of variations in the rigidity coefficient, the viscous damping coefficient, or the dry friction coefficient.

Special attention should be here drawn to works by Peterka and his coworkers, Nordmark, Chin et al., Ivanov, Shaw, and by Shaw and Holmes. Peterka et al. [32-34, 75] determine the regions of stable periodic motions and show a transition to chaotic motions both for the case of a linear, elastic-damping supporting structure, and for the case where dry friction occurs in the system. They present the results of numerical investigations in the form of time functions, phase trajectories, and Poincare maps. Nordmark [29] conducted the investigations on a motion of the onedegree-of-freedom oscillator in which impacts occur under the influence of external excitation. He undertook a trial to describe the moment of impact and showed that a change in external parameters could lead to qualitative changes in the motion character, from a periodic motion, through bifurcation cycles, up to chaotic motions. Moreover, using analytical methods, he presented a new impact phenomenon of the grazing type and a new type of bifurcation that follows from it. Various types of grazing bifurcations were discussed by Chin et al. [7]. The grazing type impacts were dealt with by Ivanov $([17,18])$ as well. Shaw and Holmes [3739] discuss a simple conservative system with one limiting stop. On the assumption that the coefficient of restitution equals zero, the authors reduce the considerations to a onedimensional map whose analysis shows that stable orbits exist for nearly all frequencies of the excitation force. Shaw [36] observed harmonic, subharmonic, and chaotic motions while analyzing the linear model of an oscillator with two limiting stops subjected to harmonic excitation, in which the processes occurring in the system at the instant of impact are modeled with Newton's hypothesis (Goldsmith [79]).

In many studies (e.g., $[4,19,26,49,80-85])$, the impact is modeled as an abrupt change in rigidity. It allows one to use various analytical methods in the dynamical analysis of the system. For instance, Blankenship and Kahraman [4] as well as Kahraman and Singh [19] analyzed a vibroimpact 
system with clearances subjected to parametric and periodic excitation. The results of the numerical analyses conducted on the basis of the methods of the harmonics balance were confirmed by the experimental results. Kim and Noah [80] employed the harmonics balance method connected with a Fourier transform procedure to analyze the system dynamics with linear characteristics in segments.

The literature devoted to mechanical systems with impacts on two degrees-of-freedom is much less extensive. Although, in this case, the first studies were published slightly later, interesting applications were already mentioned in them. Special attention should be paid to Sadek [71], Masri [86], Masri and Caughey [70], Masri and Ibrahim [73], Araki et al. [87], Bapat and Sankar [88], Cempel [5], Peterka [32, 33], Chatterjee et al. [72], Bazhenov et al. [55], Peterka and Blazejczyk-Okolewska [75], including theoretical, experimental, and numerical analyses of impact vibration dampers, Karpenko et al. ([89-91]), providing extensive analytical, numerical, and experimental studies of a Jeffcott rotor with a snubber ring, and Koizumi [92], Park [93], and Luo et al. [60], describing the applications of impact oscillators as models of moulding machines. Numerous examples of other applications of systems with impacts can be found in A. E. Kobrinskii and A. Kobrinskii [20], Babickii [47], and Jerrelind and Stensson [94].

Lately the efforts of researchers investigating the dynamics of vibroimpact systems have been focused on the theory of stability, bifurcations, the reasons for occurrence of chaos (e.g., Aidanpää and Gupta [51], Brach [95], Chen [96], Chin et al. [7], Blazejczyk-Okolewska and Kapitaniak [76, 77], Czolczynski [57], Czolczynski and Kapitaniakt [58], Di Bernardo et al. [9], Han et al. [67], Ivanov [18], Awrejcewicz and Lamarque [97], Bishop [98], Blazejczyk-Okolewska et al. [99], Brogliato [100], Guckenheimer and Holmes [101], Ibrahim [102], Rand and Moon [103], and Thompson and Bishop [104]), and control of such systems (e.g., Awrejcewicz et al. [2], Gutiérrez and Arrowsmith [105], de Souza and Caldas [66], de Souza et al. [106], Lee and Yan [23], Luo [107], Luo and Lv [62], Zhao and Wang [108], and Zinjade and Mallik [109]).

In the literature survey presented here, first of all the publications devoted to new dynamic behaviors have been mentioned. The occurrence of specific behaviors of the system is strictly related to the physical model assumed for its analysis. In Blazejczyk-Okolewska et al. [110], numerous schemes of models of mechanical systems with impacts were presented. For the initial analysis of the above-mentioned systems, physical models in which all linear and nonlinear supporting structures are described with the most elementary rheological model, a spring with linear characteristics, can be used.

The comparative studies of physical models of vibroimpact systems used in scientific considerations have led to the determination of assumptions and principles of classification of mechanical systems with impacts in which models are rigid bodies that can move along a straight line without a possibility to rotate (Blazejczyk-Okolewska et al. [110]) and to the development of the method that allows one to determine structural patterns of all such systems with an arbitrary number of degrees-of-freedom (Blazejczyk-Okolewska [111]). By a structural pattern of the mechanical system with impacts is understood a certain series of systems characterized by a specified structure of component elements (a definite configuration of fenders and connections). All structural patterns of mechanical systems constitute a set in which a kind of the connection (a spring or a damper), its character (linearity or nonlinearity), and the way the impact phenomenon is modeled are not differentiating parameters.

In the present study, the above-mentioned method has been illustrated on the example of one- and two-degrees-offreedom systems. All structural patterns of mechanical oneand two-degrees-of-freedom systems with impacts, with arbitrary connections, have been identified and described with this method. Next, structural patterns have been assigned to vibroimpact systems selected from the literature. The knowledge of all systems with impacts of a given number of degrees-of-freedom allows us to state which types have been already analyzed and which have not been investigated yet. The proposed classification of mechanical systems with impacts according to their structure allows us to rearrange the knowledge on systems with impacts and is the basis for understanding the sources of their diversity. Providing a full set of objects to be analyzed, it gives hints for new ideas and directions in designing technical devices.

In the considerations below, the terminology, concepts, and notations introduced by Blazejczyk-Okolewska [111] have been used.

\section{Systems with Two Degrees-of-Freedom}

Systems with two degrees-of-freedom $(n=2)$ have one connectedness zone. Numerous equivalent combinations and many disconnected systems can be identified among them.

The maximal number of springs (spring connections) $s=3\left(k_{1}\right.$ is the spring connecting the subsystem of a mass $m_{1}$ with the frame, $k_{12}$ the spring connecting the subsystem of a mass $m_{1}$ with the subsystem of the mass $m_{2}$, and $k_{2}$ the spring connecting the subsystem of a mass $m_{2}$ with the frame). The number of possible spring combinations is $i_{s}=8$. All connected and disconnected combinations are presented in Figures 1 and 2.

The spring connectedness zone is located between the subsystems with the masses $m_{1}$ and $m_{2}$. This zone consists of just one spring $k_{12}$, which decides whether the basic spring system will be divided into two subsystems or not (in the case of a system with a higher number of degrees-of-freedom, a division into two or more subsystems can occur, of course). The number $i_{s-s}$ of all connected spring combinations is equal to a product of the number of possible arrangements of the springs that decide about connectedness (one) and the number of possible arrangements beyond the spring connectedness zone of the remaining 0,1 , or 2 springs (four); that is to say, $i_{s-s}=1 \times 4=4$ (see Figure 1). The notations of connected spring combinations $\left(\mathbf{S}_{1}, \mathbf{S}_{2}, \mathbf{S}_{5}\right.$, and $\left.\mathbf{S}_{\mathbf{6}}\right)$ have been introduced according to the generation procedure presented 

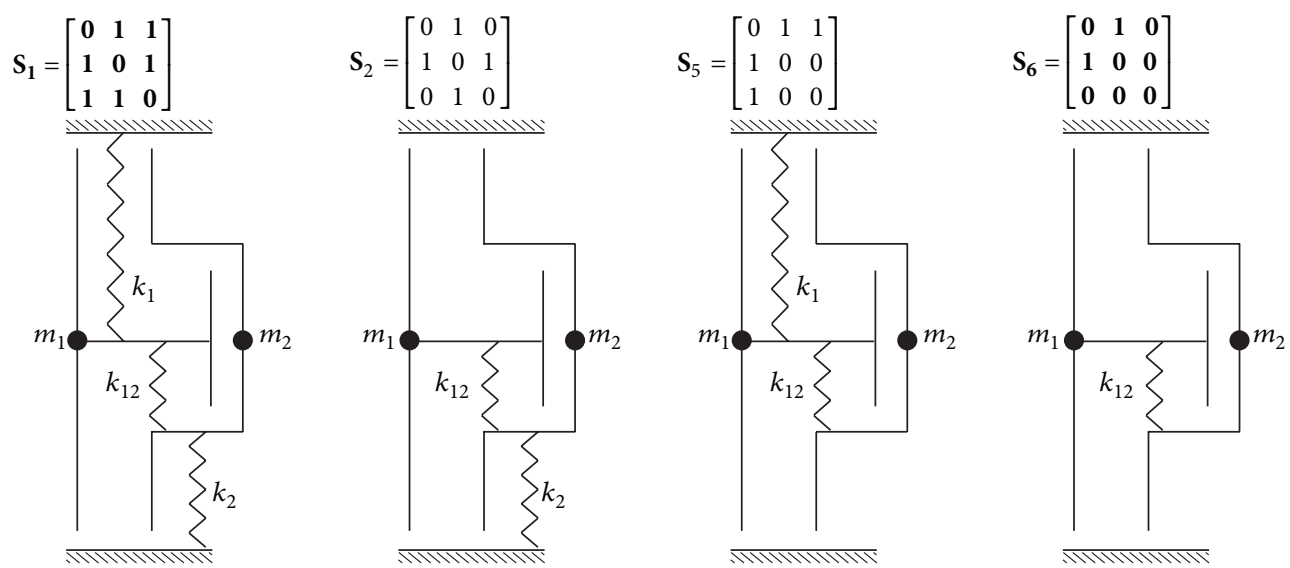

Figure 1: Connected spring combinations for $n=2$.
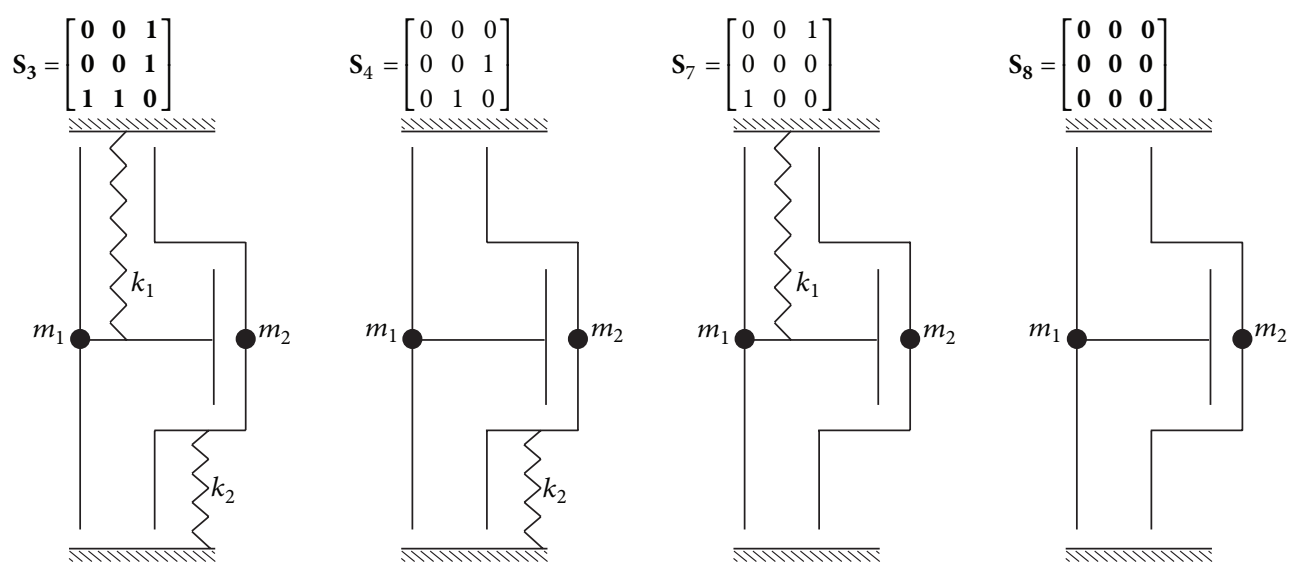

FIGURE 2: Disconnected spring combinations for $n=2$.

by Blazejczyk-Okolewska ([111, Subsection 4.1.1]). One of these systems $\left(\mathbf{S}_{1}\right)$ is the basic spring system.

Similarly, the number $i_{s-n}$ of all disconnected spring combinations can be defined. This number is equal to a product of the number of possible arrangements of the springs that decide about the connectedness (one) and the number of possible arrangements beyond the spring connectedness zone of the remaining 0,1 , or 2 springs (four); that is to say, $i_{s-n}=$ $1 \times 4=4$ (see Figure 2). The notations of disconnected spring combinations $\left(\mathbf{S}_{3}, \mathbf{S}_{4}, \mathbf{S}_{7}\right.$, and $\mathbf{S}_{8}$ ) have been introduced according to the above-mentioned generation procedure. The total number of connected and disconnected spring combinations is of course equal to the number of all spring combinations; that is, $i_{s}=i_{s-s}+i_{s-n}=4+4=8$.

In the case of connected spring combinations (Figure 1), the body of a mass $m_{1}$ acts on the body of a mass $m_{2}$ (and vice versa, of course), due to an occurrence of the spring connection between these bodies (the spring $k_{12}$ ). On the other hand, in the case of disconnected spring combinations (Figure 2), the body of a mass $m_{1}$ will never affect the behavior of the body of a mass $m_{2}$ (and vice versa, of course). A lack of the spring connection results in the fact that the bodies "will not know" about each other.
Spring adjacency matrices are attributed to all spring combinations. To list them, also the generation procedure has been used. Let us pay attention to the fact that elements and indices of some spring adjacency matrices have been bolded (in Figure 1: $\mathbf{S}_{1}, \mathbf{S}_{6}$; in Figure 2: $\mathbf{S}_{3}, \mathbf{S}_{\mathbf{8}}$ ). This bolding refers to spring systems that are equivalent to each other by inversion (the definition of equivalency has been given by BlazejczykOkolewska [111]).

We will conduct a similar analysis for impact connections. The maximal number of fenders (impact connections) is equal to $z=6$. These are the following connections: $z_{1 g}$ is the upper impact connection of the subsystem of a mass $m_{1}$ with the frame, $z_{1 d}$ the lower impact connection of the subsystem of a mass $m_{1}$ with the frame, $z_{12 g}$ the upper impact connection of the subsystem of a mass $m_{1}$ with the subsystem of a mass $m_{2}, z_{12 d}$ the lower impact connection of the subsystem of a mass $m_{1}$ with the subsystem of a mass $m_{2}, z_{2 g}$-upper impact connection of the subsystem of a mass $m_{2}$ with the frame, and $z_{2 d}$ the lower impact connection of the subsystem of a mass $m_{2}$ with the frame. The number of impact connections is equal to $i_{z}=64$. All connected and disconnected impact combinations are presented in Figures $3,4,5$, and 6 . 

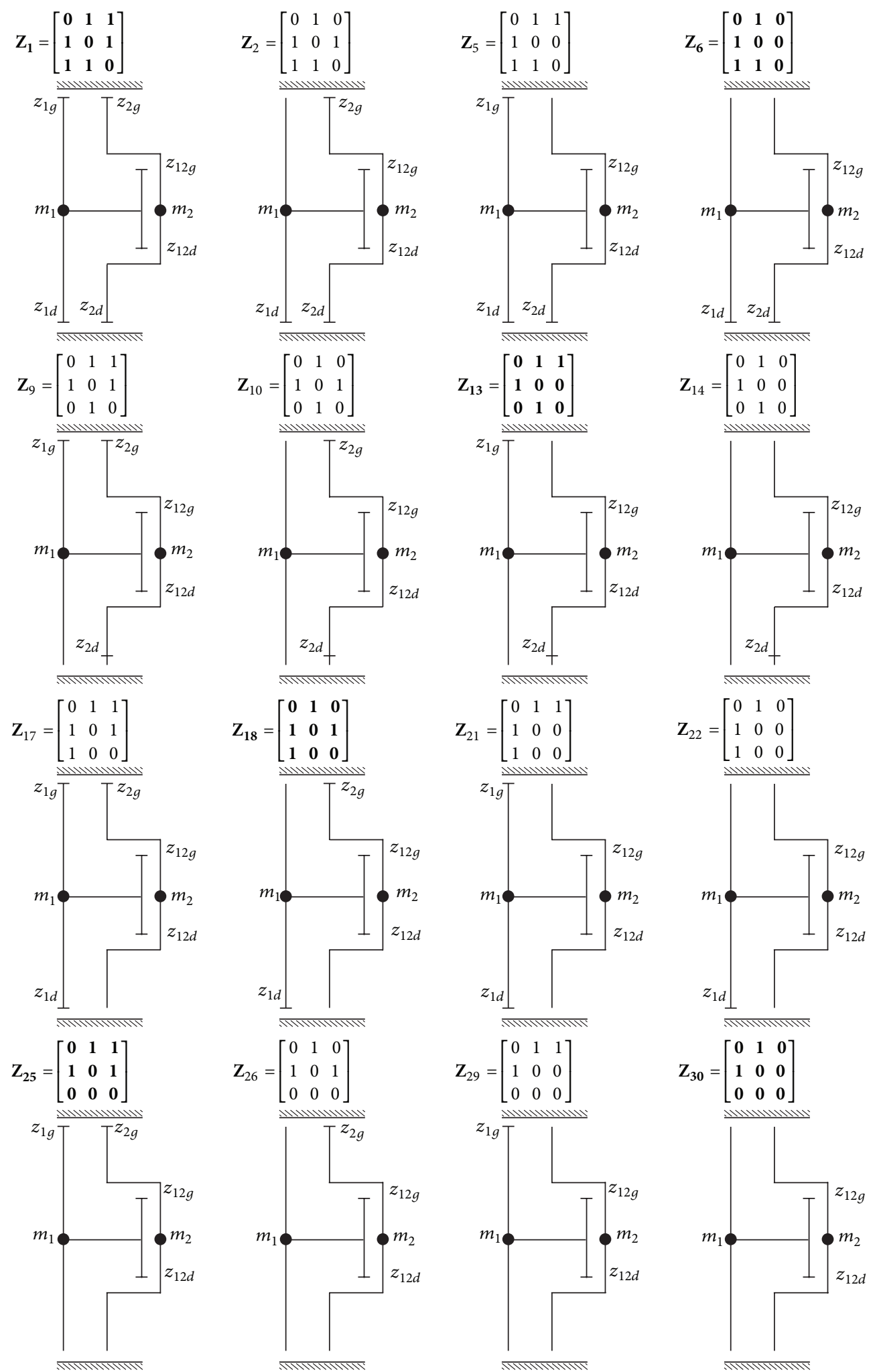

FIGURE 3: Connected impact combinations (fenders $z_{12 g}$ and $z_{12 d}$ ) for $n=2$. 

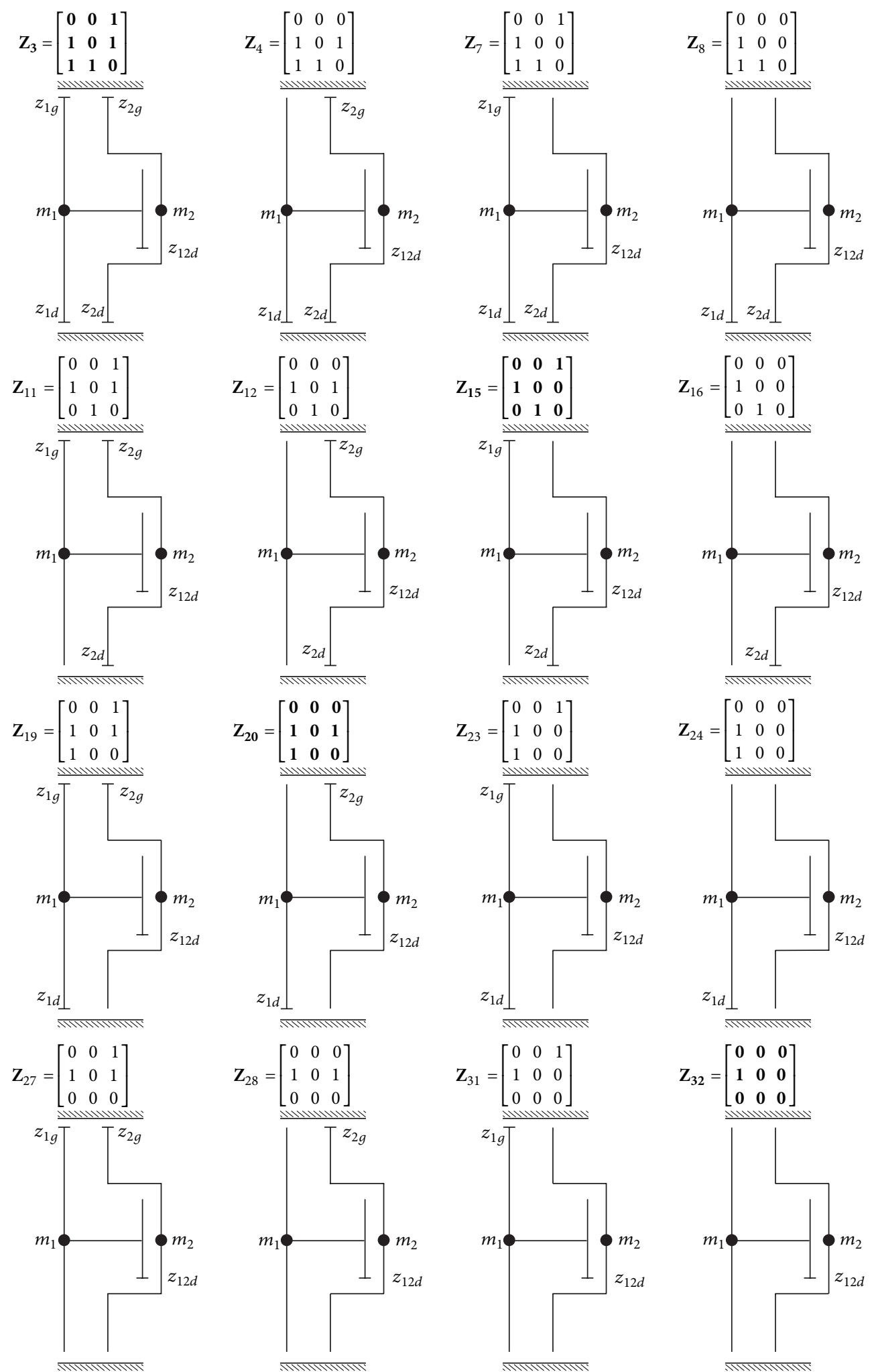

FIGURE 4: Connected impact combinations (fender $z_{12 d}$ ) for $n=2$. 

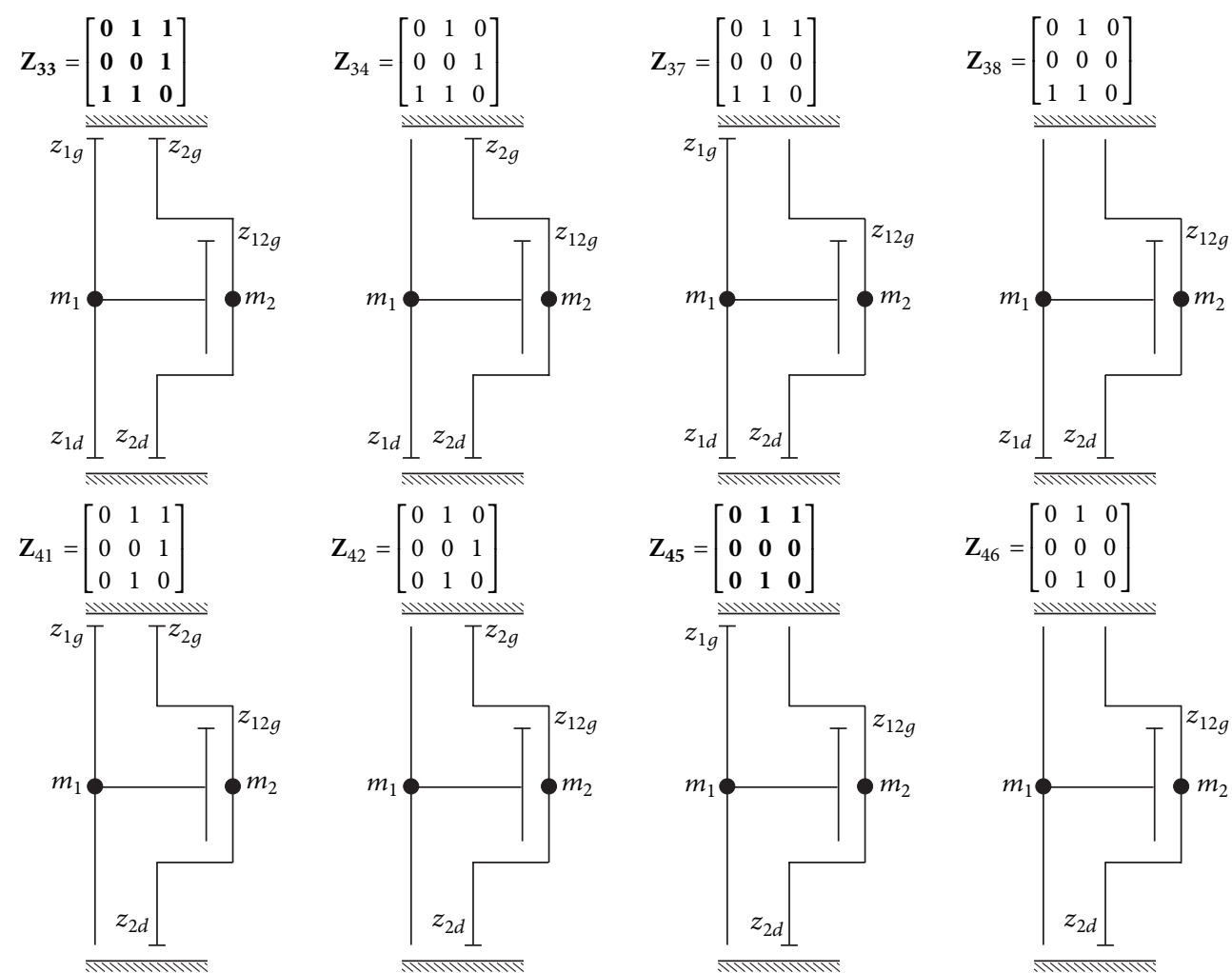

$\mathbf{Z}_{46}=\left[\begin{array}{lll}0 & 1 & 0 \\ 0 & 0 & 0 \\ 0 & 1 & 0\end{array}\right]$
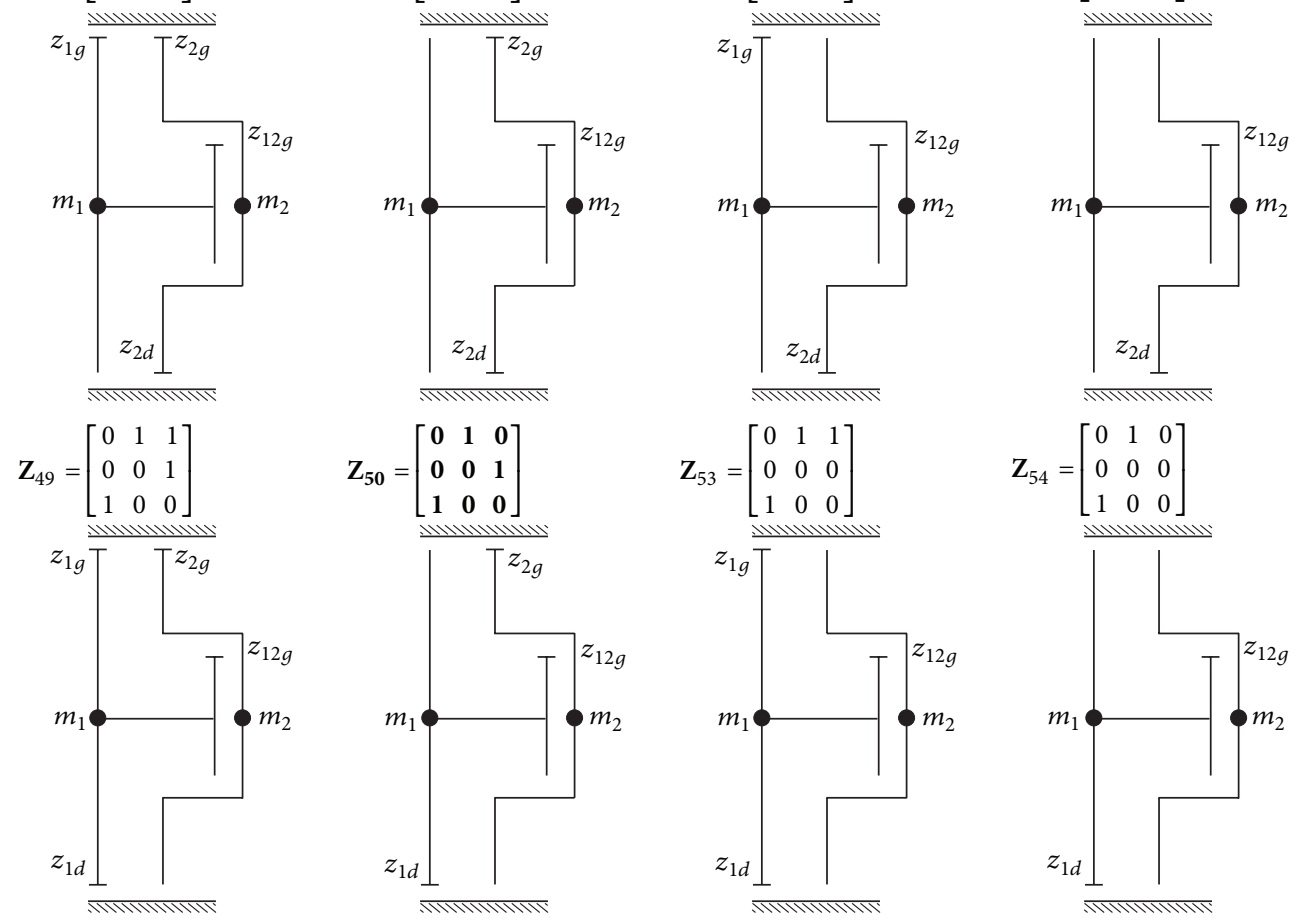

$\mathbf{Z}_{57}=\left[\begin{array}{lll}0 & 1 & 1 \\ 0 & 0 & 1 \\ 0 & 0 & 0\end{array}\right]$
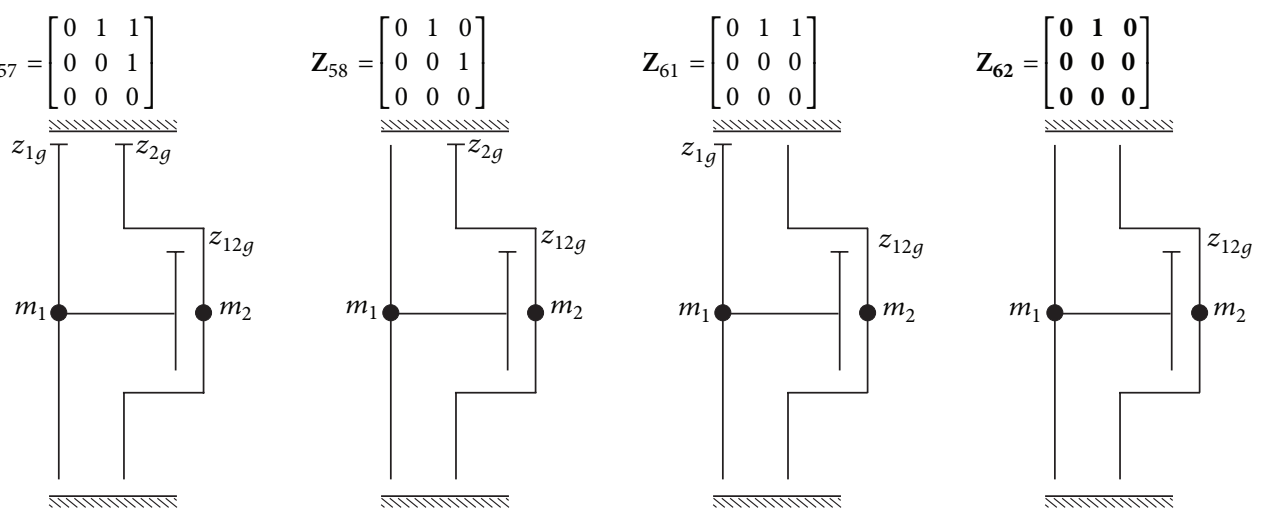

Figure 5: Connected impact combinations (fender $z_{12 g}$ ) for $n=2$. 

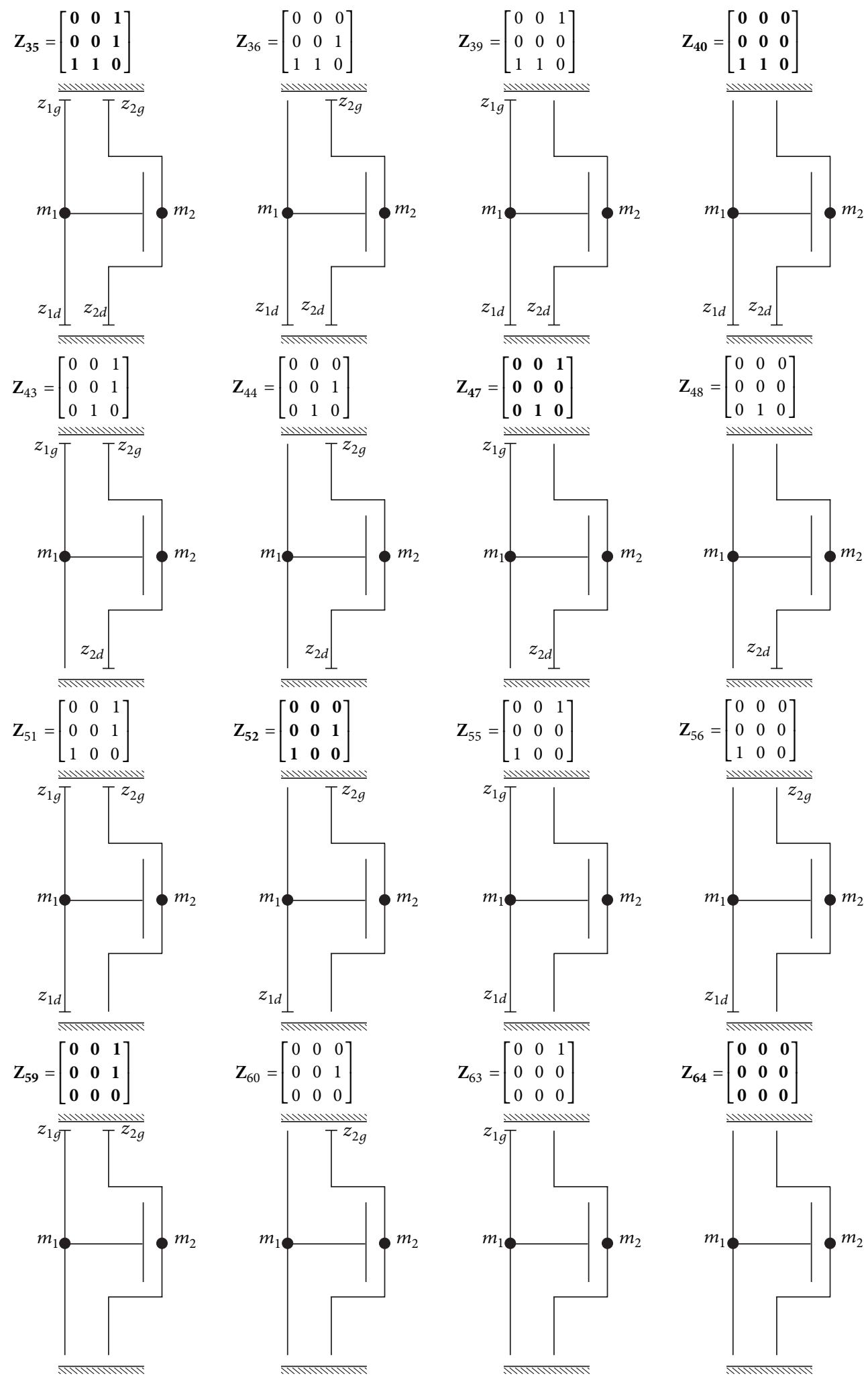

FIGURE 6: Disconnected impact combinations (lack of the fenders $z_{12 g}$ and $z_{12 d}$ ) for $n=2$. 
TABLE 1: The way connected and disconnected spring-impact combinations are generated (before elimination) for $n=2$.

\begin{tabular}{|c|c|c|}
\hline & $i_{s-s}=4:$ connected spring combinations & $\begin{array}{c}i_{s-n}=4: \text { disconnected spring } \\
\text { combinations }\end{array}$ \\
\hline $\begin{array}{l}i_{z-s}=48: \text { connected impact } \\
\text { combinations }\end{array}$ & $\begin{aligned} i_{\mathrm{s} z-s}= & i_{s-s} \times i_{z-s}=4 \times 48=192: \text { connected } \\
& \text { spring-impact combinations }\end{aligned}$ & $\begin{array}{c}i_{s z-s}=i_{s-n} \times i_{z-s}=4 \times 48=192: \\
\text { connected spring-impact combinations }\end{array}$ \\
\hline $\begin{array}{l}i_{z-n}=16: \text { disconnected } \\
\text { impact combinations }\end{array}$ & $\begin{aligned} i_{\mathrm{s} z-s}= & i_{s-s} \times i_{z-n}=4 \times 16=64: \text { connected } \\
& \text { spring-impact combinations }\end{aligned}$ & $\begin{array}{c}i_{\mathrm{sz-n}}=i_{s-n} \times i_{z-n}=4 \times 16=64: \\
\text { disconnected spring-impact } \\
\text { combinations }\end{array}$ \\
\hline
\end{tabular}

TABLE 2: Selected classes of spring-impact relations and their representatives.

\begin{tabular}{|c|c|c|c|}
\hline \multicolumn{4}{|c|}{ Classes of spring-impact relations } \\
\hline $\begin{array}{l}\text { Representatives of the } \\
\text { class of relations }\end{array}$ & $\begin{array}{l}\text { Remaining systems from } \\
\text { the class of relations }\end{array}$ & $\begin{array}{l}\text { Representatives of the } \\
\text { class of relations }\end{array}$ & $\begin{array}{l}\text { Remaining systems from } \\
\text { the class of relations }\end{array}$ \\
\hline $\mathrm{S}_{1} \mathrm{Z}_{1}$ & & $\mathrm{~S}_{5} \mathrm{Z}_{1}$ & $\mathrm{~S}_{2} \mathrm{Z}_{1}$ \\
\hline $\mathbf{S}_{1} \mathbf{Z}_{17}$ & $\mathbf{S}_{1} \mathbf{Z}_{2}, \mathbf{S}_{1} \mathbf{Z}_{9}, \mathbf{S}_{1} \mathbf{Z}_{5}$ & $\mathbf{S}_{5} \mathbf{Z}_{9}$ & $\mathbf{S}_{5} \mathbf{Z}_{2}, \mathbf{S}_{2} \mathbf{Z}_{5}, \mathbf{S}_{2} \mathbf{Z}_{17}$ \\
\hline$S_{1} Z_{18}$ & $\mathbf{S}_{1} \mathbf{Z}_{13}$ & $\mathbf{S}_{5} \mathbf{Z}_{10}$ & $\mathbf{S}_{2} \mathbf{Z}_{21}$ \\
\hline $\mathbf{S}_{1} \mathbf{Z}_{21}$ & $\mathbf{S}_{1} \mathbf{Z}_{10}$ & $\mathbf{S}_{5} \mathbf{Z}_{17}$ & $\mathbf{S}_{2} \mathbf{Z}_{2}, \mathbf{S}_{2} \mathbf{Z}_{9}, \mathbf{S}_{5} \mathbf{Z}_{5}$ \\
\hline $\mathrm{S}_{1} \mathrm{Z}_{25}$ & $S_{1} Z_{6}$ & $\mathbf{S}_{5} \mathbf{Z}_{18}$ & $S_{2} Z_{13}, S_{2} Z_{18}, S_{5} Z_{13}$ \\
\hline $\mathbf{S}_{1} \mathbf{Z}_{29}$ & $\mathbf{S}_{1} \mathbf{Z}_{14}, \mathbf{S}_{1} \mathbf{Z}_{26}, \mathbf{S}_{1} \mathbf{Z}_{22}$ & $\mathbf{S}_{5} \mathbf{Z}_{21}$ & $\mathbf{S}_{2} \mathbf{Z}_{10}$ \\
\hline $\mathrm{S}_{1} \mathrm{Z}_{30}$ & & $\mathbf{S}_{5} \mathbf{Z}_{25}$ & $\mathbf{S}_{2} \mathbf{Z}_{6}, \mathbf{S}_{2} \mathbf{Z}_{25}, \mathbf{S}_{5} \mathbf{Z}_{6}$ \\
\hline $\mathrm{S}_{1} \mathrm{Z}_{33}$ & $\mathrm{~S}_{1} \mathrm{Z}_{3}$ & $\mathbf{S}_{5} \mathbf{Z}_{26}$ & $\mathbf{S}_{5} \mathbf{Z}_{14}, \mathbf{S}_{2} \mathbf{Z}_{22}, \mathbf{S}_{2} \mathbf{Z}_{29}$ \\
\hline $\mathrm{S}_{1} \mathrm{Z}_{35}$ & & $\mathbf{S}_{5} \mathbf{Z}_{29}$ & $S_{2} Z_{14}, S_{2} Z_{26}, S_{5} Z_{22}$ \\
\hline $\mathbf{S}_{1} \mathbf{Z}_{41}$ & $\mathbf{S}_{1} \mathbf{Z}_{4}, \mathbf{S}_{1} \mathbf{Z}_{37}, \mathbf{S}_{1} \mathbf{Z}_{19}$ & $\mathbf{S}_{5} \mathbf{Z}_{30}$ & $\mathbf{S}_{2} \mathbf{Z}_{30}$ \\
\hline $\mathrm{S}_{1} \mathrm{Z}_{45}$ & $\mathbf{S}_{1} \mathbf{Z}_{20}$ & $\mathbf{S}_{5} \mathbf{Z}_{33}$ & $\mathbf{S}_{2} \mathbf{Z}_{3}, \mathbf{S}_{2} \mathbf{Z}_{33}, \mathbf{S}_{5} \mathbf{Z}_{3}$ \\
\hline $\mathbf{S}_{1} \mathbf{Z}_{49}$ & $\mathbf{S}_{1} \mathbf{Z}_{7}, \mathbf{S}_{1} \mathbf{Z}_{34}, \mathrm{~S}_{1} \mathbf{Z}_{11}$ & $\mathbf{S}_{5} \mathbf{Z}_{34}$ & $\mathbf{S}_{2} \mathbf{Z}_{7}, \mathbf{S}_{2} \mathbf{Z}_{49}, \mathbf{S}_{5} \mathbf{Z}_{11}$ \\
\hline $\mathrm{S}_{1} \mathrm{Z}_{50}$ & $\mathbf{S}_{1} \mathbf{Z}_{15}$ & $\mathbf{S}_{5} \mathbf{Z}_{35}$ & $\mathbf{S}_{2} \mathbf{Z}_{35}$ \\
\hline $\mathbf{S}_{1} \mathbf{Z}_{51}$ & $\mathbf{S}_{1} \mathbf{Z}_{36}, \mathbf{S}_{1} \mathbf{Z}_{43}, \mathbf{S}_{1} \mathbf{Z}_{39}$ & $\mathbf{S}_{5} \mathbf{Z}_{37}$ & $\mathbf{S}_{2} \mathbf{Z}_{4}, \mathbf{S}_{2} \mathbf{Z}_{41}, \mathbf{S}_{5} \mathbf{Z}_{19}$ \\
\hline $\mathrm{S}_{1} \mathrm{Z}_{52}$ & $\mathbf{S}_{1} \mathbf{Z}_{47}$ & $\mathbf{S}_{5} \mathbf{Z}_{38}$ & $\mathbf{S}_{2} \mathbf{Z}_{8}, \mathbf{S}_{2} \mathbf{Z}_{57}, \mathbf{S}_{5} \mathbf{Z}_{27}$ \\
\hline $\mathrm{S}_{1} \mathrm{Z}_{53}$ & $S_{1} Z_{12}, S_{1} Z_{42}, S_{1} Z_{23}$ & $\mathbf{S}_{5} \mathbf{Z}_{41}$ & $S_{5} Z_{4}, S_{2} Z_{37}, S_{2} Z_{19}$ \\
\hline $\mathbf{S}_{1} \mathbf{Z}_{55}$ & $\mathbf{S}_{1} \mathbf{Z}_{44}$ & $\mathbf{S}_{5} \mathbf{Z}_{42}$ & $S_{5} Z_{12}, S_{2} Z_{53}, S_{2} Z_{23}$ \\
\hline $\mathbf{S}_{1} \mathbf{Z}_{57}$ & $S_{1} Z_{8}, S_{1} Z_{38}, S_{1} Z_{27}$ & $\mathbf{S}_{5} \mathbf{Z}_{43}$ & $S_{5} Z_{36}, S_{2} Z_{39}, S_{2} Z_{51}$ \\
\hline $\mathbf{S}_{1} \mathbf{Z}_{58}$ & $S_{1} Z_{16}, S_{1} Z_{54}, S_{1} Z_{31}$ & $\mathbf{S}_{5} \mathbf{Z}_{44}$ & $\mathbf{S}_{2} \mathbf{Z}_{55}$ \\
\hline$S_{1} Z_{59}$ & $\mathbf{S}_{1} \mathbf{Z}_{40}$ & $\mathbf{S}_{5} \mathbf{Z}_{45}$ & $\mathbf{S}_{2} \mathbf{Z}_{20}, \mathbf{S}_{2} \mathbf{Z}_{45}, \mathbf{S}_{5} \mathbf{Z}_{20}$ \\
\hline $\mathbf{S}_{1} \mathbf{Z}_{61}$ & $\mathbf{S}_{1} \mathbf{Z}_{24}, \mathbf{S}_{1} \mathbf{Z}_{46}, \mathbf{S}_{1} \mathbf{Z}_{28}$ & $\mathbf{S}_{5} \mathbf{Z}_{46}$ & $\mathbf{S}_{2} \mathbf{Z}_{24}, \mathbf{S}_{2} \mathbf{Z}_{61}, \mathbf{S}_{5} \mathbf{Z}_{28}$ \\
\hline$S_{1} Z_{62}$ & $\mathbf{S}_{1} \mathbf{Z}_{32}$ & $\mathbf{S}_{5} \mathbf{Z}_{49}$ & $\mathbf{S}_{5} \mathbf{Z}_{7}, \mathbf{S}_{2} \mathbf{Z}_{34}, \mathbf{S}_{2} \mathbf{Z}_{11}$ \\
\hline $\mathbf{S}_{1} \mathbf{Z}_{63}$ & $\mathbf{S}_{1} \mathbf{Z}_{48}, \mathbf{S}_{1} \mathbf{Z}_{60}, \mathbf{S}_{1} \mathbf{Z}_{56}$ & $\mathrm{~S}_{5} \mathrm{Z}_{50}$ & $S_{2} Z_{15}, S_{2} Z_{50}, S_{5} Z_{15}$ \\
\hline \multirow{13}{*}{$S_{1} Z_{64}$} & & $\mathbf{S}_{5} \mathbf{Z}_{51}$ & $\mathbf{S}_{2} \mathbf{Z}_{36}, \mathbf{S}_{2} \mathbf{Z}_{43}, \mathbf{S}_{5} \mathbf{Z}_{39}$ \\
\hline & & $\mathbf{S}_{5} \mathbf{Z}_{52}$ & $S_{2} Z_{47}, S_{2} Z_{52}, S_{5} Z_{47}$ \\
\hline & & $\mathbf{S}_{5} \mathbf{Z}_{53}$ & $\mathbf{S}_{2} \mathbf{Z}_{12}, \mathbf{S}_{2} \mathbf{Z}_{42}, \mathbf{S}_{5} \mathbf{Z}_{23}$ \\
\hline & & $\mathbf{S}_{5} \mathbf{Z}_{54}$ & $\mathbf{S}_{2} \mathbf{Z}_{16}, \mathbf{S}_{2} \mathbf{Z}_{58}, \mathbf{S}_{5} \mathbf{Z}_{31}$ \\
\hline & & $\mathbf{S}_{5} \mathbf{Z}_{55}$ & $\mathrm{~S}_{2} \mathrm{Z}_{44}$ \\
\hline & & $\mathbf{S}_{5} \mathbf{Z}_{57}$ & $\mathbf{S}_{5} \mathbf{Z}_{8}, \mathbf{S}_{2} \mathbf{Z}_{38}, \mathbf{S}_{2} \mathbf{Z}_{27}$ \\
\hline & & $\mathbf{S}_{5} \mathbf{Z}_{58}$ & $S_{5} Z_{16}, S_{2} Z_{54}, S_{2} Z_{31}$ \\
\hline & & $\mathbf{S}_{5} \mathbf{Z}_{59}$ & $\mathbf{S}_{2} \mathbf{Z}_{40}, \mathbf{S}_{2} \mathbf{Z}_{59}, \mathbf{S}_{5} \mathbf{Z}_{40}$ \\
\hline & & $\mathbf{S}_{5} \mathbf{Z}_{60}$ & $S_{5} Z_{48}, S_{2} Z_{56}, S_{2} Z_{63}$ \\
\hline & & $\mathbf{S}_{5} \mathbf{Z}_{61}$ & $\mathbf{S}_{5} \mathbf{Z}_{24}, \mathbf{S}_{2} \mathbf{Z}_{46}, \mathbf{S}_{2} \mathbf{Z}_{28}$ \\
\hline & & $\mathbf{S}_{5} \mathbf{Z}_{62}$ & $S_{2} Z_{32}, S_{2} Z_{62}, S_{5} Z_{32}$ \\
\hline & & $\mathbf{S}_{5} \mathbf{Z}_{63}$ & $\mathbf{S}_{2} \mathbf{Z}_{48}, \mathbf{S}_{2} \mathbf{Z}_{60}, \mathbf{S}_{5} \mathbf{Z}_{56}$ \\
\hline & & $\mathbf{S}_{5} \mathbf{Z}_{64}$ & $\mathbf{S}_{2} \mathbf{Z}_{64}$ \\
\hline
\end{tabular}


TABLE 3: Table of spring relations for $n=2$.

Fields of spring information

\begin{tabular}{lc}
$\mathbf{S}_{i}$ & $\mathbf{S}_{i}^{P}$ \\
\hline $\mathbf{1}$ & $\mathbf{1}$ \\
2 & 5 \\
$\mathbf{3}$ & 5 \\
4 & $\mathbf{3}$ \\
5 & 7 \\
$\mathbf{6}$ & 2 \\
7 & $\mathbf{6}$ \\
$\mathbf{8}$ & 4 \\
\hline
\end{tabular}

The impact connectedness zone is located between the subsystems of masses $m_{1}$ and $m_{2}$. This zone consists of two fenders $z_{12 g}$ and $z_{12 d}$ that decide whether a division of the basic impact system into two subsystems will occur or not. The number $i_{z-s}$ of all connected impact combinations is equal to a product of the number of possible arrangements of the impact connections that decide about the connectedness (three) and the number of possible arrangements, beyond the impact adjacency zone, of the remaining impact connections $\left(2^{4}=16\right)$; that is to say, $i_{z-s}=3 \times 16=48$. Figure 3 shows the systems: $\mathbf{Z}_{1}, \mathbf{Z}_{2}, \mathbf{Z}_{5}, \mathbf{Z}_{\mathbf{6}}, \mathbf{Z}_{9}, \mathbf{Z}_{10}, \mathbf{Z}_{13}, \mathbf{Z}_{14}, \mathbf{Z}_{17}$, $\mathbf{Z}_{\mathbf{1 8}}, \mathbf{Z}_{21}, \mathbf{Z}_{22}, \mathbf{Z}_{\mathbf{2 5}}, \mathbf{Z}_{26}, \mathbf{Z}_{29}$, and $\mathbf{Z}_{\mathbf{3 0}}$, that is, all such connected impact combinations that the connection between subsystems occurs both via the upper fender $z_{12 \mathrm{~g}}$ and via the lower fender $z_{12 \mathrm{~d}}$. One of these systems $\left(\mathbf{Z}_{\mathbf{1}}\right)$ is the basic impact system. In Figure 4 the following systems are marked: $\mathbf{Z}_{3}, \mathbf{Z}_{4}, \mathbf{Z}_{7}, \mathbf{Z}_{8}, \mathbf{Z}_{11}, \mathbf{Z}_{12}, \mathbf{Z}_{15}, \mathbf{Z}_{16}, \mathbf{Z}_{19}, \mathbf{Z}_{\mathbf{2 0}}, \mathbf{Z}_{23}, \mathbf{Z}_{24}, \mathbf{Z}_{27}, \mathbf{Z}_{28}$, $\mathbf{Z}_{31}$, and $\mathbf{Z}_{32}$, that is, all connected impact combinations in which the connection between subsystems occurs only via the lower fender $z_{12 \mathrm{~d}}$. Figure 5 presents the systems: $\mathbf{Z}_{33}, \mathbf{Z}_{34}$, $\mathbf{Z}_{37}, \mathbf{Z}_{38}, \mathbf{Z}_{41}, \mathbf{Z}_{42}, \mathbf{Z}_{45}, \mathbf{Z}_{46}, \mathbf{Z}_{49}, \mathbf{Z}_{50}, \mathbf{Z}_{53}, \mathbf{Z}_{54}, \mathbf{Z}_{57}, \mathbf{Z}_{58}, \mathbf{Z}_{61}$, and $\mathbf{Z}_{62}$, that is, all connected impact combinations in which the connection between subsystems occurs only via the upper fender $z_{12 \mathrm{~g}}$. The notations of connected impact combinations have been introduced according to the generation procedure discussed by Blazejczyk-Okolewska ([111, Section 4.1.1]). The number $i_{z-n}$ of all disconnected impact combinations is equal to a product of the number of possible arrangements of the impact connections that decide about the disconnectedness (one) and the number of possible arrangements, beyond the impact connectedness zone, of the remaining impact connections $\left(2^{4}=16\right)$; that is to say, $i_{z-n}=1 \times 16=16$. In Figure 6, the following systems are depicted: $\mathbf{Z}_{35}, \mathbf{Z}_{36}, \mathbf{Z}_{39}$, $\mathbf{Z}_{40}, \mathbf{Z}_{43}, \mathbf{Z}_{44}, \mathbf{Z}_{47}, \mathbf{Z}_{48}, \mathbf{Z}_{51}, \mathbf{Z}_{52}, \mathbf{Z}_{55}, \mathbf{Z}_{56}, \mathbf{Z}_{59}, \mathbf{Z}_{60}, \mathbf{Z}_{63}$, and $\mathbf{Z}_{\mathbf{6 4}}$, that is, all disconnected impact combinations. The total number of connected and disconnected impact combinations is equal to the number of all impact combinations $i_{z}=i_{z-s}+$ $i_{z-n}=48+16=64$. The impact adjacency matrices are attributed to all impact combinations.

Let us notice that the elements and indices of some impact adjacency matrices, similarly as spring adjacency matrices, have been bolded (in Figure $3: \mathbf{Z}_{1}, \mathbf{Z}_{\mathbf{6}}, \mathbf{Z}_{\mathbf{1 3}}, \mathbf{Z}_{\mathbf{1 8}}, \mathbf{Z}_{\mathbf{2 5}}$, and $\mathbf{Z}_{\mathbf{3 0}}$; in Figure $4: \mathbf{Z}_{3}, \mathbf{Z}_{15}, \mathbf{Z}_{\mathbf{2 0}}$, and $\mathbf{Z}_{32}$; in Figure 5: $\mathbf{Z}_{33}, \mathbf{Z}_{\mathbf{4 5}}, \mathbf{Z}_{50}$,
TABLE 4: Table of impact relations for $n=2$.

Fields of impact information

\begin{tabular}{cccc}
$\mathbf{Z}_{i}$ & $\mathbf{Z}_{i}^{T}$ & $\mathbf{Z}_{i}^{P}$ & $\mathbf{Z}_{i}$ \\
\hline $\mathbf{1}$ & 1 & $\mathbf{1}$ & \\
2 & 9 & 5 & \\
$\mathbf{3}$ & 33 & 33 \\
4 & 41 & 37 & \\
5 & 17 & 2 & \\
$\mathbf{6}$ & 25 & $\mathbf{6}$ & \\
7 & 49 & 34 & \\
8 & 57 & 38 \\
9 & 2 & 17
\end{tabular}

10

11

12

13 
TABLE 4: Continued.

\begin{tabular}{lccc}
\hline \multicolumn{4}{c}{ Fields of impact information } \\
$\mathbf{Z}_{i}$ & $\mathbf{Z}_{i}^{T}$ & $\mathbf{Z}_{i}^{P}$ & $\mathbf{Z}_{i}^{P T}$ \\
\hline 46 & 28 & 24 & 61 \\
$\mathbf{4 7}$ & 52 & 52 & $\mathbf{4 7}$ \\
48 & 60 & 56 & 63 \\
49 & 7 & 11 & 34 \\
$\mathbf{5 0}$ & 15 & 15 & $\mathbf{5 0}$ \\
51 & 39 & 43 & 36 \\
$\mathbf{5 2}$ & 47 & 47 & $\mathbf{5 2}$ \\
53 & 23 & 12 & 42 \\
54 & 31 & 16 & 58 \\
55 & 55 & 44 & 44 \\
56 & 63 & 48 & 60 \\
57 & 8 & 27 & 38 \\
58 & 16 & 31 & 54 \\
$\mathbf{5 9}$ & 40 & $\mathbf{5 9}$ & 40 \\
60 & 48 & 63 & 56 \\
61 & 24 & 28 & 46 \\
$\mathbf{6 2}$ & 32 & 32 & $\mathbf{6 2}$ \\
63 & 56 & 60 & 48 \\
$\mathbf{6 4}$ & 64 & $\mathbf{6 4}$ & $\mathbf{6 4}$ \\
\hline
\end{tabular}

and $\mathbf{Z}_{62}$; in Figure 6: $\mathbf{Z}_{35}, \mathbf{Z}_{40}, \mathbf{Z}_{47}, \mathbf{Z}_{52}, \mathbf{Z}_{59}$, and $\mathbf{Z}_{64}$ ). These are all impact systems equivalent to each other via inversion or translocation.

In the case of forty-eight connected impact combinations (Figures 3, 4, and 5), the body of a mass $m_{1}$ acts on the body of a mass $m_{2}$ due to an occurrence of the impact connection between the subsystems (fenders $z_{12 \mathrm{~g}}$ and $z_{12 \mathrm{~d}}$ in Figure 3, one of these fenders in Figures 4 and 5). On the other hand, in the case of sixteen disconnected impact combinations (Figure 6), the body of a mass $m_{1}$ will never affect the behavior of the body of a mass $m_{2}$ (and vice versa, of course). A lack of the impact connection (a lack of both fenders $z_{12 \mathrm{~g}}$ and $z_{12 \mathrm{~d}}$ ) will cause that the bodies "will not know" about each other.

According to the assumed principle (Phase I, BlazejczykOkolewska [111]), we will match now each spring combination with each impact combination. The total number of connected spring-impact combinations (for $n=2$ before elimination) is equal to $i_{s z-s}=448$, whereas the total number of disconnected spring-impact combinations (before elimination) is equal to $i_{s z-n}=64$. Table 1 shows Phase $I$ for the system of $n=2$. As a result of matching all connected spring combinations (Figure 1) with all connected impact combinations (Figures 3, 4, and 5), connected spring-impact combinations arise. Their total number equals $i_{s z-s}=i_{s-s} \times$ $i_{z-s}=4 \times 48=192$. As a result of matching all connected spring combinations (Figure 1) with all disconnected impact combinations (Figure 6), connected spring-impact combinations arise. Their total number is $i_{s z-s}=i_{s-s} \times i_{z-n}=4 \times 16=64$. As a result of matching all disconnected spring combinations (Figure 2) with all connected impact combinations (Figures 3, 4 , and 5), connected spring-impact combinations arise. Their number is equal to $i_{s z-s}=i_{s-n} \times i_{z-s}=4 \times 48=192$. As a result of matching all disconnected spring combinations (Figure 2) with all disconnected impact combinations (Figure 6), disconnected spring-impact combinations arise. Their number equals $i_{s z-n}=i_{s-n} \times i_{z-n}=4 \times 16=64$. The number of connected spring-impact combinations added to the number of disconnected spring-impact combinations is equal to the number of all spring-impact combinations; that is to say, $i_{s z}=i_{s z-s}+i_{s z-n}=448+64=512$.

Due to the convenience of presentation, spring, impact and spring-impact combinations will be referred to as spring, impact, and spring-impact systems up to the end of this section.

The next stage consists in the elimination of equivalent combinations (subphase I of Phase II, Blazejczyk-Okolewska [111]). For $n=2$, it can be conducted without a computer, but it is a very time-consuming procedure.

Let us begin with the generation of the adjacency matrix of all spring-impact systems. We take the first one, and we generate a transposed, inverted, and translocated matrix to it. Next, we check the equivalency of the taken matrix to itself and to the adjacency matrices of the subsequent spring-impact systems. If any equivalency is identified (see equations $(2) \div(4)$, Blazejczyk-Okolewska [111]), we make a class of relations, and we choose the system with the highest member as its representative (see the principles of selection, Blazejczyk-Okolewska [111]). The above-mentioned activities should be repeated for each spring-impact system which has not been included in any class of relations yet.

As an example, let us consider the spring-impact system $\mathbf{S}_{2} \mathbf{Z}_{23}$. It is equivalent to other systems $\mathbf{S}_{2} \mathbf{Z}_{53} \mathbf{S}_{5} \mathbf{Z}_{12}$ and $\mathbf{S}_{5} \mathbf{Z}_{42}$. The class of relations in this case has four elements. We choose $\mathbf{S}_{5} \mathbf{Z}_{42}$ as its representative as it is the system with the highest number, and the systems $\mathbf{S}_{2} \mathbf{Z}_{23}, \mathbf{S}_{2} \mathbf{Z}_{53}$, and $\mathbf{S}_{5} \mathbf{Z}_{12}$ are thus eliminated.

Table 2 presents a list of selected classes of relations and their representatives. The first two columns include all springimpact systems that arise as a result of matching the spring system $\mathbf{S}_{1}$ with all impact systems $\mathbf{Z}_{1} \div \mathbf{Z}_{64}$. An analogous list can be given for the remaining spring systems equivalent to one another via inversion, that is, $\mathbf{S}_{3}, \mathbf{S}_{\mathbf{6}}$, and $\mathbf{S}_{\mathbf{8}}$ (in Table 2, we replace $S_{1}$ by the subsequent systems $S_{3}, S_{6}$, and $S_{8}$ ). The last two columns in the table include all spring-impact systems that result from matching the spring system $\boldsymbol{S}_{5}$ with all impact systems $\mathbf{Z}_{1} \div \mathbf{Z}_{64}$. An analogous list can be prepared for the spring system $\mathbf{S}_{7}$ (in Table 2, we replace $\boldsymbol{S}_{5}$ by the spring system $S_{7}$ and $S_{2}$ by the spring system $\mathbf{S}_{4}$ ). For $n=2$, there are 168 classes of relations. Among them, we can distinguish 24 classes of relations in which spring-impact systems are disconnected (see Table 5 discussed further in this section as well). Classes of spring-impact relations can be oneelement (one spring-impact system which is simultaneously the representative of the class of relations), two elements (two equivalent spring-impact systems and one of them is the representative of the class of relations), or four elements (four equivalent spring-impact systems and one of them is the representative of the class of relations).

Below, in the context of the two-degrees-of-freedom system, an application of the procedures developed by 
TABLE 5: List of connected and disconnected representatives of classes of connection-impact (spring-impact) relations.

\begin{tabular}{|c|c|c|c|c|c|c|c|c|c|c|c|c|c|c|c|c|}
\hline \multicolumn{17}{|c|}{ Connected representatives of classes of relations } \\
\hline \multirow{3}{*}{$P_{1}=S_{1}$} & $\begin{array}{r}\mathrm{Z}_{1} \\
1\end{array}$ & $\begin{array}{c}\mathbf{Z}_{17} \\
4\end{array}$ & $\begin{array}{c}\mathrm{Z}_{18} \\
2\end{array}$ & $\begin{array}{c}\mathbf{Z}_{21} \\
2\end{array}$ & $\begin{array}{c}\mathrm{Z}_{25} \\
2\end{array}$ & $\begin{array}{c}\mathbf{Z}_{29} \\
4\end{array}$ & $\begin{array}{c}\mathrm{Z}_{30} \\
1\end{array}$ & & & & & & & & & \\
\hline & $\begin{array}{c}Z_{33} \\
2\end{array}$ & $\begin{array}{c}\mathbf{Z}_{41} \\
4\end{array}$ & $\begin{array}{c}\mathrm{Z}_{45} \\
2\end{array}$ & $\begin{array}{r}\mathrm{Z}_{49} \\
4\end{array}$ & $\mathrm{Z}_{50}$ & $\begin{array}{r}Z_{53} \\
4\end{array}$ & $\begin{array}{r}\mathbf{Z}_{57} \\
4\end{array}$ & $\begin{array}{c}\mathrm{Z}_{58} \\
4\end{array}$ & $\begin{array}{c}\mathrm{Z}_{61} \\
4\end{array}$ & $\begin{array}{c}\mathrm{Z}_{62} \\
2\end{array}$ & & & & & & \\
\hline & $\begin{array}{c}\mathrm{Z}_{35} \\
1\end{array}$ & $\begin{array}{c}\mathbf{Z}_{51} \\
4\end{array}$ & $\begin{array}{c}\mathrm{Z}_{52} \\
2\end{array}$ & $\begin{array}{c}\mathbf{Z}_{55} \\
2\end{array}$ & $\begin{array}{c}Z_{59} \\
2\end{array}$ & $\begin{array}{c}\mathrm{Z}_{63} \\
4\end{array}$ & $\begin{array}{c}\mathrm{Z}_{64}{ }^{*} \\
1\end{array}$ & & & & & & & & & \\
\hline \multirow[t]{2}{*}{$P_{3}=S_{3}$} & $\begin{array}{r}\mathrm{Z}_{1} \\
\mathbf{1}\end{array}$ & $\begin{array}{c}\mathbf{Z}_{17} \\
4\end{array}$ & $\begin{array}{c}\mathrm{Z}_{18} \\
2\end{array}$ & $\begin{array}{c}\mathbf{Z}_{21} \\
2\end{array}$ & $\begin{array}{c}\mathrm{Z}_{25} \\
2\end{array}$ & $\begin{array}{c}\mathbf{Z}_{29} \\
4\end{array}$ & $\begin{array}{c}\mathrm{Z}_{30} \\
1\end{array}$ & & & & & & & & & \\
\hline & $\begin{array}{c}\mathrm{Z}_{33} \\
2\end{array}$ & $\begin{array}{c}\mathbf{Z}_{41} \\
4\end{array}$ & $\begin{array}{c}\mathrm{Z}_{45} \\
\mathbf{2}\end{array}$ & $\begin{array}{c}\mathbf{Z}_{49} \\
4\end{array}$ & $\begin{array}{c}Z_{50} \\
2\end{array}$ & $\begin{array}{c}\mathrm{Z}_{53} \\
4\end{array}$ & $\begin{array}{c}\mathbf{Z}_{57} \\
4\end{array}$ & $\begin{array}{c}\mathbf{Z}_{58} \\
4\end{array}$ & $\begin{array}{c}\mathrm{Z}_{61} \\
4\end{array}$ & $\begin{array}{c}\mathrm{Z}_{62} \\
2\end{array}$ & & & & & & \\
\hline \multirow{3}{*}{$\mathbf{P}_{5}=\mathbf{S}_{5}$} & $\begin{array}{r}\mathbf{Z}_{1} \\
2\end{array}$ & $\begin{array}{r}Z_{9} \\
4\end{array}$ & $\begin{array}{c}\mathbf{Z}_{10} \\
2\end{array}$ & $\begin{array}{r}\mathrm{Z}_{17} \\
4\end{array}$ & $\begin{array}{c}\mathrm{Z}_{18} \\
4\end{array}$ & $\begin{array}{c}\mathbf{Z}_{21} \\
2\end{array}$ & $\begin{array}{c}\mathrm{Z}_{25} \\
4\end{array}$ & $\begin{array}{c}\mathrm{Z}_{26} \\
4\end{array}$ & $\begin{array}{c}\mathbf{Z}_{29} \\
4\end{array}$ & $\begin{array}{c}\mathbf{Z}_{30} \\
2\end{array}$ & & & & & & \\
\hline & $\begin{array}{c}\mathrm{Z}_{33} \\
4\end{array}$ & $\begin{array}{c}\mathrm{Z}_{34} \\
4\end{array}$ & $\begin{array}{c}\mathrm{Z}_{37} \\
4\end{array}$ & $\begin{array}{c}\mathrm{Z}_{38} \\
4\end{array}$ & $\begin{array}{c}\mathbf{Z}_{41} \\
4\end{array}$ & $\begin{array}{c}\mathbf{Z}_{42} \\
4\end{array}$ & $\begin{array}{c}\mathrm{Z}_{45} \\
4\end{array}$ & $\begin{array}{c}\mathrm{Z}_{46} \\
4\end{array}$ & $\begin{array}{c}\mathbf{Z}_{49} \\
4\end{array}$ & $\begin{array}{c}\mathbf{Z}_{50} \\
4\end{array}$ & $\begin{array}{c}Z_{53} \\
4\end{array}$ & $\begin{array}{c}\mathrm{Z}_{54} \\
4\end{array}$ & $\begin{array}{c}\mathbf{Z}_{57} \\
4\end{array}$ & $\begin{array}{c}\mathrm{Z}_{58} \\
4\end{array}$ & $\begin{array}{c}\mathbf{Z}_{61} \\
4\end{array}$ & $\begin{array}{c}\mathrm{Z}_{62} \\
4\end{array}$ \\
\hline & $\begin{array}{c}Z_{35} \\
2 \\
\end{array}$ & $\begin{array}{c}\mathbf{Z}_{43} \\
4 \\
\end{array}$ & $\begin{array}{c}\mathbf{Z}_{44} \\
2 \\
\end{array}$ & $\begin{array}{c}Z_{51} \\
4 \\
\end{array}$ & $\begin{array}{c}Z_{52} \\
4 \\
\end{array}$ & $\begin{array}{c}Z_{55} \\
2 \\
\end{array}$ & $\begin{array}{c}\mathrm{Z}_{59} \\
4 \\
\end{array}$ & $\begin{array}{c}\mathrm{Z}_{60} \\
4 \\
\end{array}$ & $\begin{array}{c}\mathrm{Z}_{63} \\
4\end{array}$ & $\mathrm{Z}_{64}{ }^{*}$ & & & & & & \\
\hline \multirow{3}{*}{$P_{6}=S_{6}$} & $\begin{array}{r}\mathrm{Z}_{1} \\
1\end{array}$ & $\begin{array}{c}\mathrm{Z}_{17} \\
4\end{array}$ & $\begin{array}{c}\mathrm{Z}_{18} \\
2\end{array}$ & $\begin{array}{c}\mathbf{Z}_{21} \\
2\end{array}$ & $\begin{array}{c}Z_{25} \\
2\end{array}$ & $\begin{array}{c}\mathbf{Z}_{29} \\
4\end{array}$ & $\begin{array}{c}\mathrm{Z}_{30} \\
1\end{array}$ & & & & & & & & & \\
\hline & $\begin{array}{c}\mathrm{Z}_{33} \\
2\end{array}$ & $\begin{array}{c}\mathbf{Z}_{41} \\
4\end{array}$ & $\begin{array}{c}\mathrm{Z}_{45} \\
2\end{array}$ & $\begin{array}{c}\mathbf{Z}_{49} \\
4\end{array}$ & $\begin{array}{c}Z_{50} \\
2\end{array}$ & $\begin{array}{c}\mathbf{Z}_{53} \\
4\end{array}$ & $\begin{array}{c}\mathbf{Z}_{57} \\
4\end{array}$ & $\begin{array}{c}\mathbf{Z}_{58} \\
4\end{array}$ & $\mathrm{Z}_{61}$ & $\begin{array}{c}\mathrm{Z}_{62} \\
2\end{array}$ & & & & & & \\
\hline & $\begin{array}{c}\mathrm{Z}_{35} \\
1\end{array}$ & $\begin{array}{c}\mathbf{Z}_{51} \\
4\end{array}$ & $\begin{array}{c}Z_{52} \\
2\end{array}$ & $\begin{array}{c}Z_{55} \\
2\end{array}$ & $\begin{array}{c}Z_{59} \\
2\end{array}$ & $\begin{array}{c}\mathrm{Z}_{63} \\
4\end{array}$ & $\mathrm{Z}_{64}{ }^{*}$ & & & & & & & & & \\
\hline \multirow[t]{2}{*}{$\mathbf{P}_{7}=\mathbf{S}_{7}$} & $\begin{array}{r}\mathbf{Z}_{1} \\
2\end{array}$ & $\begin{array}{r}\mathbf{Z}_{9} \\
4\end{array}$ & $\begin{array}{c}\mathbf{Z}_{10} \\
2\end{array}$ & $\begin{array}{r}\mathrm{Z}_{17} \\
4\end{array}$ & $\begin{array}{c}\mathrm{Z}_{18} \\
4\end{array}$ & $\begin{array}{c}\mathbf{Z}_{21} \\
2\end{array}$ & $\begin{array}{c}\mathrm{Z}_{25} \\
4\end{array}$ & $\begin{array}{c}\mathbf{Z}_{26} \\
4\end{array}$ & $\begin{array}{c}\mathbf{Z}_{29} \\
4\end{array}$ & $\begin{array}{c}\mathbf{Z}_{30} \\
\mathbf{2}\end{array}$ & & & & & & \\
\hline & $\begin{array}{c}\mathrm{Z}_{33} \\
4\end{array}$ & $\begin{array}{c}\mathbf{Z}_{34} \\
4\end{array}$ & $\begin{array}{c}\mathrm{Z}_{37} \\
4\end{array}$ & $\begin{array}{c}\mathrm{Z}_{38} \\
4\end{array}$ & $\begin{array}{c}\mathbf{Z}_{41} \\
4\end{array}$ & $\begin{array}{c}\mathrm{Z}_{42} \\
4\end{array}$ & $\begin{array}{c}\mathrm{Z}_{45} \\
4\end{array}$ & $\begin{array}{c}\mathbf{Z}_{46} \\
4\end{array}$ & $\begin{array}{c}\mathbf{Z}_{49} \\
4\end{array}$ & $\begin{array}{c}\mathbf{Z}_{50} \\
4\end{array}$ & $\begin{array}{c}\mathbf{Z}_{53} \\
4\end{array}$ & $\mathbf{Z}_{54}$ & $\begin{array}{c}\mathbf{Z}_{57} \\
4\end{array}$ & $\begin{array}{c}\mathrm{Z}_{58} \\
4\end{array}$ & $\begin{array}{c}\mathbf{Z}_{61} \\
4\end{array}$ & $\begin{array}{r}\mathbf{Z}_{62} \\
4\end{array}$ \\
\hline \multirow[t]{2}{*}{$\mathbf{P}_{8}=\mathrm{S}_{8}{ }^{\#}$} & $\begin{array}{r}\mathrm{Z}_{1} \\
\mathbf{1}\end{array}$ & $\begin{array}{c}\mathbf{Z}_{17} \\
4\end{array}$ & $\begin{array}{c}\mathrm{Z}_{18} \\
2\end{array}$ & $\begin{array}{c}\mathbf{Z}_{21} \\
2\end{array}$ & $\begin{array}{c}\mathrm{Z}_{25} \\
2\end{array}$ & $\begin{array}{c}\mathrm{Z}_{29} \\
4\end{array}$ & $\begin{array}{c}\mathrm{Z}_{30} \\
\mathbf{1}\end{array}$ & & & & & & & & & \\
\hline & $\begin{array}{c}\mathrm{Z}_{33} \\
2\end{array}$ & $\begin{array}{c}\mathbf{Z}_{41} \\
4\end{array}$ & $\begin{array}{c}\mathrm{Z}_{45} \\
2\end{array}$ & $\begin{array}{c}\mathrm{Z}_{49} \\
4\end{array}$ & $\begin{array}{c}Z_{50} \\
2\end{array}$ & $\begin{array}{c}\mathrm{Z}_{53} \\
4\end{array}$ & $\begin{array}{c}\mathbf{Z}_{57} \\
4\end{array}$ & $\begin{array}{c}\mathbf{Z}_{58} \\
4\end{array}$ & $\begin{array}{r}\mathrm{Z}_{61} \\
4\end{array}$ & $\begin{array}{c}Z_{62} \\
2\end{array}$ & & & & & & \\
\hline \multicolumn{17}{|c|}{ Disconnected representatives of classes of relations } \\
\hline $\mathbf{P}_{3}=\mathbf{S}_{3}$ & $\begin{array}{c}\mathrm{Z}_{35} \\
1\end{array}$ & $\begin{array}{c}\mathbf{Z}_{51} \\
4\end{array}$ & $\begin{array}{c}\mathrm{Z}_{52} \\
2\end{array}$ & $\begin{array}{c}\mathbf{Z}_{55} \\
2\end{array}$ & $\begin{array}{c}Z_{59} \\
2\end{array}$ & $\begin{array}{c}\mathrm{Z}_{63} \\
4\end{array}$ & $\begin{array}{c}\mathrm{Z}_{64}{ }^{*} \\
1\end{array}$ & & & & & & & & & \\
\hline$P_{7}=S_{7}$ & $\begin{array}{c}\mathrm{Z}_{35} \\
2\end{array}$ & $\begin{array}{c}\mathbf{Z}_{43} \\
4\end{array}$ & $\begin{array}{c}\mathrm{Z}_{44} \\
2\end{array}$ & $\begin{array}{c}\mathrm{Z}_{51} \\
4\end{array}$ & $\begin{array}{c}\mathrm{Z}_{52} \\
4\end{array}$ & $\begin{array}{c}Z_{55} \\
2\end{array}$ & $\begin{array}{c}Z_{59} \\
4\end{array}$ & $\begin{array}{c}\mathrm{Z}_{60} \\
4\end{array}$ & $\begin{array}{r}\mathrm{Z}_{63} \\
4\end{array}$ & $\begin{array}{c}\mathrm{Z}_{64}{ }^{*} \\
2\end{array}$ & & & & & & \\
\hline $\mathbf{P}_{8}=\mathrm{S}_{8}^{\#}$ & $\begin{array}{c}\mathrm{Z}_{35} \\
1\end{array}$ & $\begin{array}{c}\mathrm{Z}_{51} \\
4\end{array}$ & $\begin{array}{c}\mathrm{Z}_{52} \\
\mathbf{2}\end{array}$ & $\begin{array}{c}Z_{55} \\
2\end{array}$ & $\begin{array}{c}Z_{59} \\
2\end{array}$ & $\begin{array}{c}\mathrm{Z}_{63} \\
4\end{array}$ & $\mathrm{Z}_{64}{ }^{*}$ & & & & & & & & & \\
\hline
\end{tabular}

${ }^{*}$ Intensively shaded.

" Slightly shaded.

Blazejczyk-Okolewska [111] will be discussed. It is worth noticing that, whenever we take the subsequent adjacency matrix, we will have the full information on equivalencies to other adjacency matrices (both for spring and impact systems).

Table 3 is a table of spring relations for $n=2$, that is to say, a list of all spring systems (the first column $\mathbf{S}_{i}$ ) together with the information on their inversed equivalency (the second column $\mathbf{S}_{i}^{P}$ ). This table has been made on the basis of the spring table generation procedure (Blazejczyk-Okolewska [111, Section 4.1.2]). Whenever we take the subsequent spring adjacency matrix, we have the full information on it. It follows from Table 3 that four spring systems are equivalent to one another via inversion $\left(\mathbf{S}_{1}, \mathbf{S}_{3}, \mathbf{S}_{\mathbf{6}}\right.$, and $\left.\mathbf{S}_{\mathbf{8}}\right)$, and there are two pairs of spring systems equivalent via inversion: $\boldsymbol{S}_{5} \sim \mathbf{S}_{2}$ and $\mathbf{S}_{7} \sim \mathbf{S}_{4}\left(5=\mathbf{S}_{5}>\mathbf{S}_{2}=2,7=\mathbf{S}_{7}>\mathbf{S}_{4}=4\right)$.

Table 4 is a table of impact relations for $n=2$, that is to say, a list of all impact systems (the first column $\mathbf{Z}_{i}$ ) with the information on their transposed (the second column $\mathbf{Z}_{i}^{T}$ ), inversed (the third column $\mathbf{Z}_{i}^{P}$ ), and translocated (the fourth column $\mathbf{Z}_{i}^{P T}$ ) equivalency. This table has been made according to the impact table generation procedure (Blazejczyk-Okolewska [111, Section 4.1.2]). Whenever we take the next impact adjacency matrix, we have the full information on it. We can read from Table 4 that for twentyfour impact systems, the number of the matrix $\mathbf{Z}_{i}$ (it refers to the lower index) fulfils condition (6) described by BlazejczykOkolewska [111]. All the systems $\left(\mathbf{Z}_{\mathbf{1}}, \mathbf{Z}_{17}, \mathbf{Z}_{\mathbf{1 8}}, \mathbf{Z}_{21}, \mathbf{Z}_{\mathbf{2 5}}\right.$, 
TABLE 6: Examples from the references $(n=1)$ and the notations of the structural pattern $\left(S_{p}\right)$ of the systems considered in them.

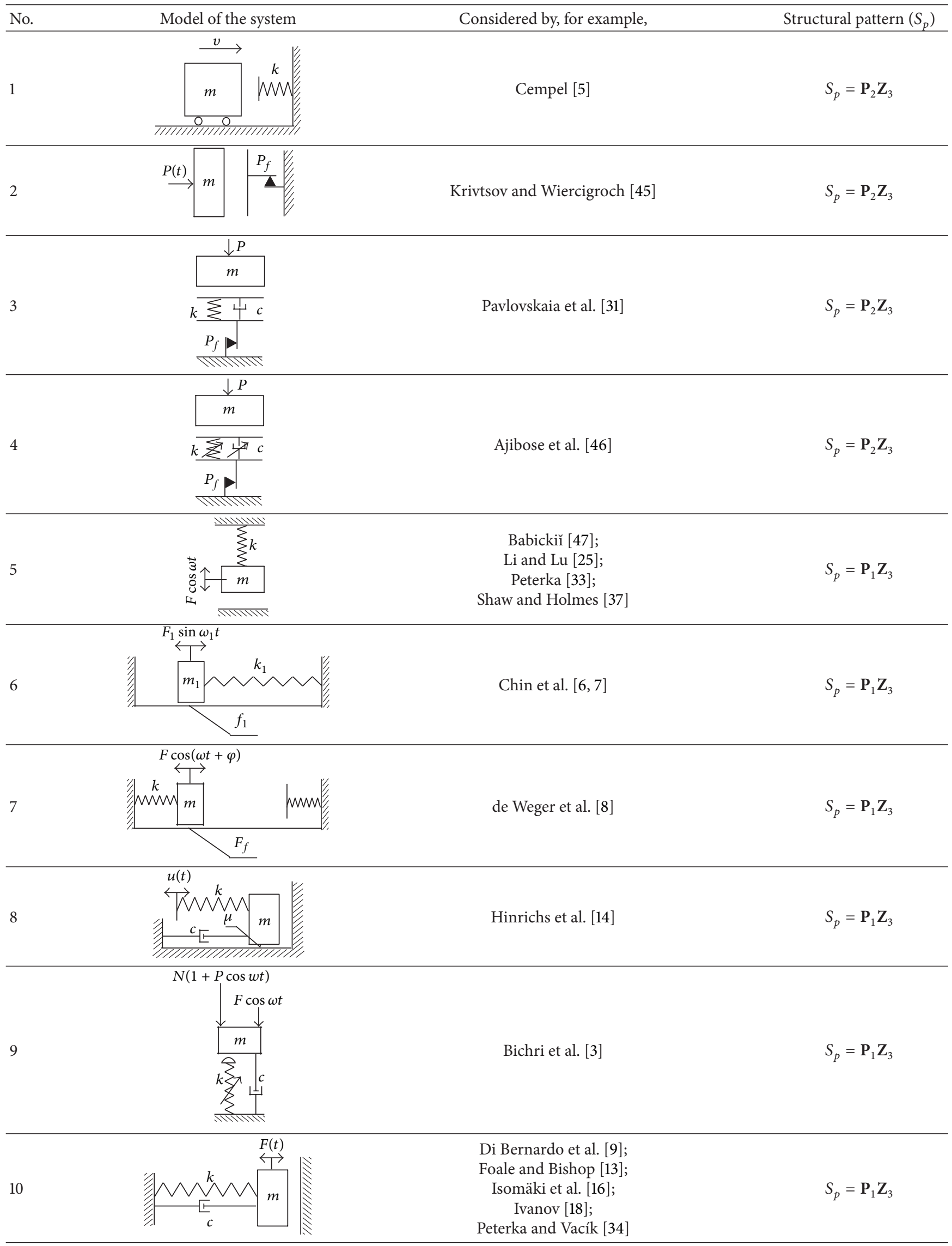


TABle 6: Continued.

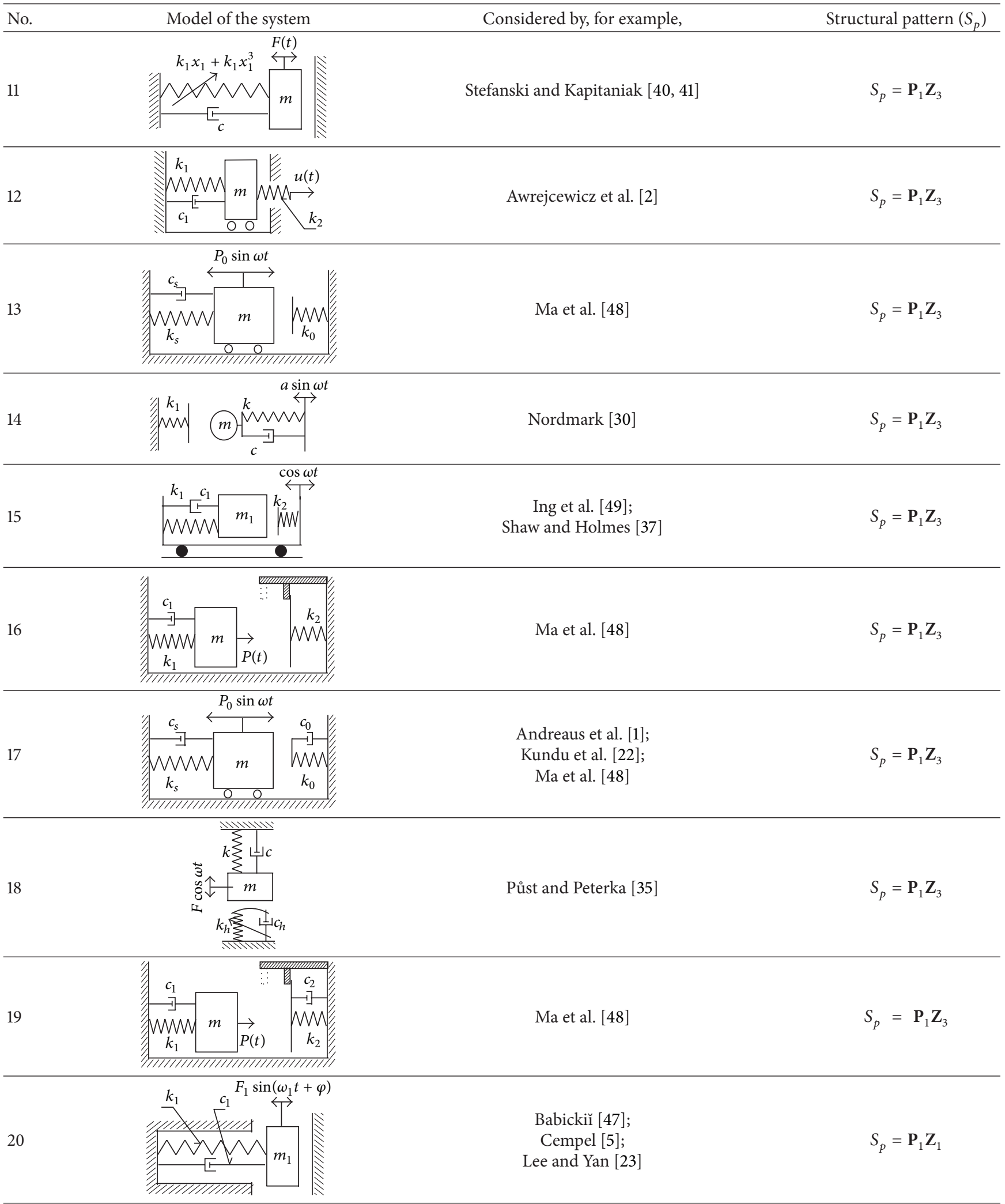


TABle 6: Continued.

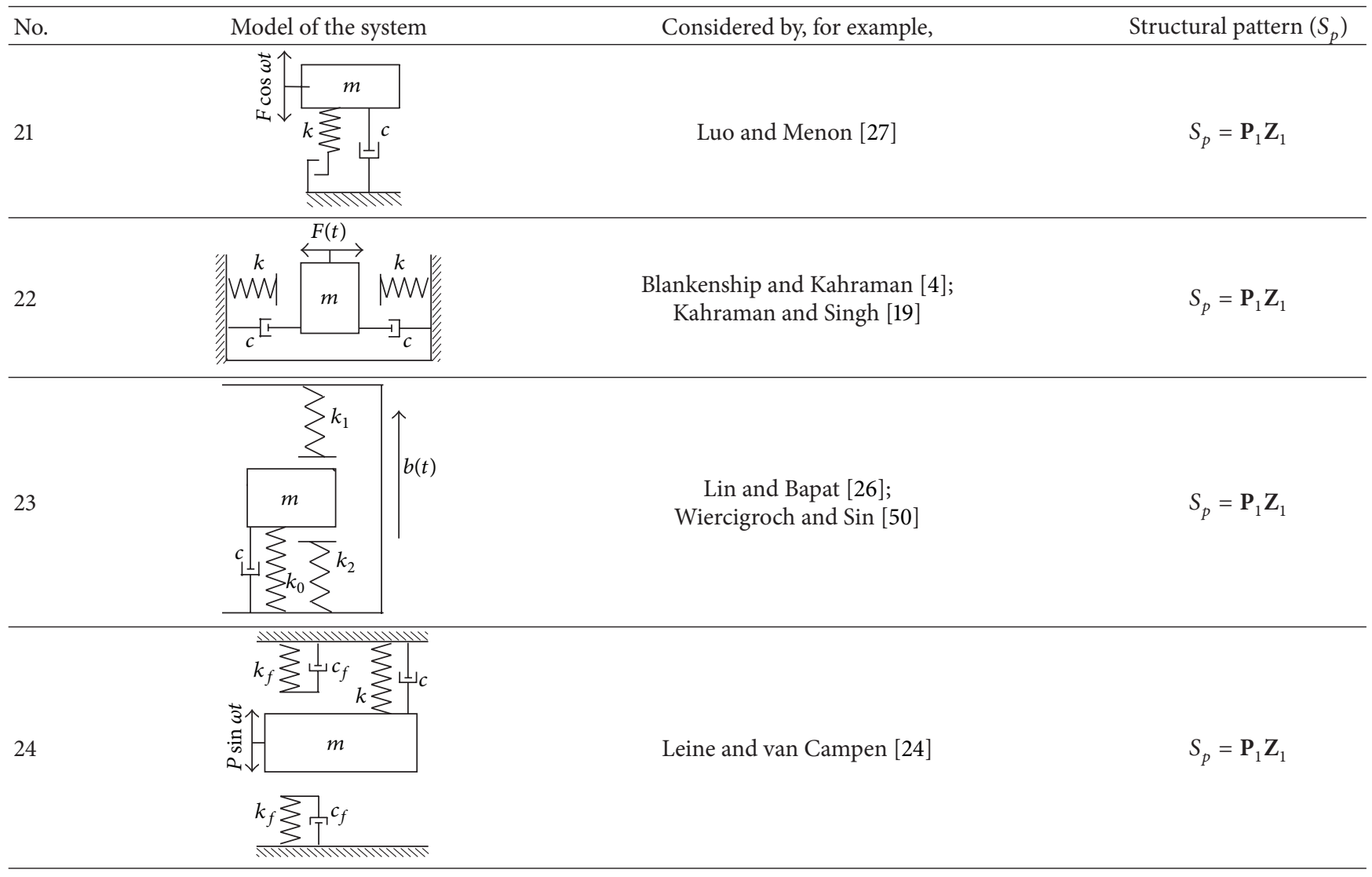

$Z_{29}, Z_{30}, Z_{33}, Z_{35}, Z_{41}, Z_{45}, Z_{49}, Z_{50}, Z_{51}, Z_{52}, Z_{53}, Z_{55}$, $\mathbf{Z}_{57}, \mathbf{Z}_{58}, \mathbf{Z}_{59}, \mathbf{Z}_{61}, \mathbf{Z}_{\mathbf{6 2}}, \mathbf{Z}_{63}$, and $\mathbf{Z}_{\mathbf{6 4}}$ ) will be matched to those spring systems which are equivalent to one another via inversion $\left(\mathbf{S}_{1}, \mathbf{S}_{3}, \mathbf{S}_{\mathbf{6}}\right.$, and $\left.\mathbf{S}_{\mathbf{8}}\right)$. After matching, we will obtain 96 spring-impact systems that are the representatives of classes of relations. Among them, 48 systems are equivalent to one another via inversion or translocation. Their lower indices will be bolded.

The remaining two spring systems $\left(\mathbf{S}_{5}\right.$ and $\left.\mathbf{S}_{7}\right)$ should be matched to impact systems fulfilling condition (6) and, additionally, to impact systems from the classes of relations that fulfill condition (7) of the numbers equal to the higher number out of the two numbers $\mathbf{Z}^{P}$ and $\mathbf{Z}^{P T}$. All the impact systems satisfying these conditions, and we have 36 such systems altogether $\left(\mathbf{Z}_{\mathbf{1}}, \mathbf{Z}_{9}, \mathbf{Z}_{10}, \mathbf{Z}_{17}, \mathbf{Z}_{\mathbf{1 8}}, \mathbf{Z}_{21}, \mathbf{Z}_{\mathbf{2 5}}, \mathbf{Z}_{26}, \mathbf{Z}_{29}\right.$, $\mathbf{Z}_{30}, \mathbf{Z}_{33}, \mathbf{Z}_{34}, \mathbf{Z}_{35}, \mathbf{Z}_{37}, \mathbf{Z}_{38}, \mathbf{Z}_{41}, \mathbf{Z}_{42}, \mathbf{Z}_{43}, \mathbf{Z}_{44}, \mathbf{Z}_{45}, \mathbf{Z}_{46}, \mathbf{Z}_{49}$, $\mathbf{Z}_{50}, \mathbf{Z}_{51}, \mathbf{Z}_{52}, \mathbf{Z}_{53}, \mathbf{Z}_{54}, \mathbf{Z}_{55}, \mathbf{Z}_{57}, \mathbf{Z}_{58}, \mathbf{Z}_{59}, \mathbf{Z}_{60}, \mathbf{Z}_{61}, \mathbf{Z}_{62}, \mathbf{Z}_{63}$, and $\left.\mathbf{Z}_{64}\right)$, after matching to the spring systems $\left(\mathbf{S}_{5}\right.$ and $\left.\mathbf{S}_{7}\right)$, yield 72 spring-impact systems that make another group of the representatives of classes of relations.

The total number of spring-impact systems which remain after the elimination of equivalent systems is thus $96+72=$ 168.

Table 5 shows all spring-impact systems that have not been eliminated. They are divided into two groups: connected systems and disconnected systems.
The list has been obtained with the identity procedures (Blazejczyk-Okolewska [111, Subsections 4.1 and 4.2]), which eliminate equivalent systems and investigate their connectedness. In the first column of Table 5 , there are spring systems. In the remaining part of the table, there are impact systems. The digit written under the symbol of the impact system corresponds to the number of systems in the given class of spring-impact relations (including the representative of this class). The bolding of this digit and of the index of the adjacency matrix $\mathbf{S}_{\mathbf{i}} \mathbf{Z}_{\mathbf{j}}$ means that the given spring-impact system is equivalent to itself via inversion of translocation. On such a list as the one prepared, an actual spring-impact system can be found easily. There are altogether 144 connected spring-impact systems (including 3 combinations without impacts), whereas the number of all disconnected springimpact systems is equal to 24 . It means that, as a result of the elimination of equivalent and disconnected systems, the original number of systems (512) has decreased more than three times (144).

Table 5 presents these spring-impact systems that have been selected as the representatives of classes of relations. Its comparison to Tables 3 and 4 provides the information on all the systems which belong to the given class of relations.

The spring-impact systems (structural patterns) presented in Table 5 are written in such a way that the given spring connection and the first line starting with $\mathbf{Z}_{1}$ correspond to the connections of the impact systems having both 

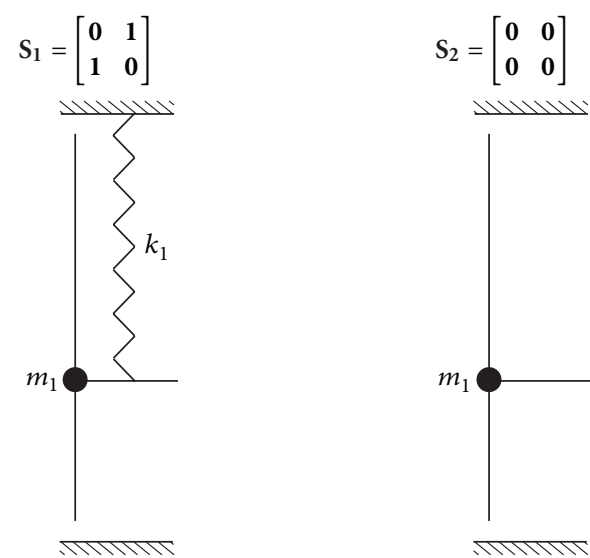

FIGURE 7: Spring combinations for $n=1$.

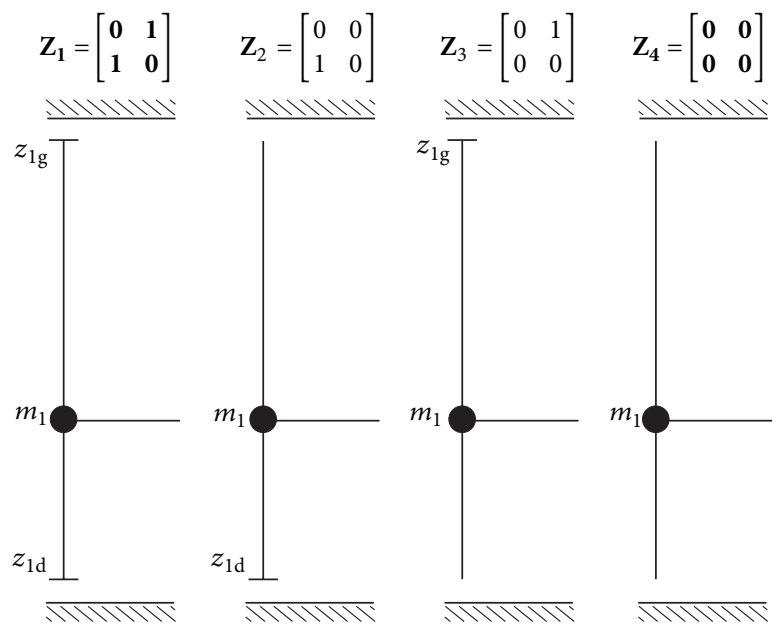

Figure 8: Impact combinations for $n=1$.

fenders in the connectedness zone, the second line beginning with $\mathbf{Z}_{33}$ to the connections of the impact systems that have one fender in the connectedness zone, and the third line starting with $\mathbf{Z}_{33}$ to the connections of the impact system that do not have fenders in the connectedness zone. The shaded cells describe systems in which there is no impact connection (the cell intensively shaded), they have no spring connection (the cell lightly shaded), or there is no impact or spring connection (connection of the cell slightly shaded to the cell very intensively shaded). Such systems will be considered in Sections 3 and 4.

One of the spring-impact systems described in Table 5, that is, $S_{1} Z_{1}$, includes the full configuration of springs and fenders (the maximum number of springs and the maximum number of fenders). It is thus the basic spring-impact system for $n=2$. All the remaining spring-impact systems can be made out of it via removing a certain number of springs or fenders.

The analysis of spring-impact systems is definitely simplified for $n=1$ and becomes much more difficult for $n \geq 3$. A classification of spring-impact systems with one degree-offreedom is presented below.

\section{Systems with One Degree-of-Freedom}

The maximum number of springs for the system with one degree-of-freedom $(n=1)$ is equal to $s=1\left(k_{1}\right.$ is the spring connecting the body of a mass $m_{1}$ with the frame, see the first system in Figure 7). The number of possible spring combinations $i_{s}=2$. In Figure 7, all combinations with their adjacency matrices are presented.

In this case it is difficult to talk about the spring connectedness (subphase II of Phase II): either the system has no spring connecting it to the frame ( $\mathbf{S}_{2}$ in Figure 7$)$, or has such a spring $\left(S_{1}\right.$ in Figure 7$)$, but it does not belong to the spring connectedness zone.

The maximum number of fenders (impact connections) for $n=1$ is $z=2\left(z_{1 g}\right.$ is the upper impact connection of the body of a mass $m_{1}$ with the frame and $z_{1 d}$ and the lower impact connection of the body of a mass $m_{1}$ with the frame). Both impact connections and their notations are marked in Figure 8 . The number of possible impact combinations $i_{z}=$ 4. Figure 8 shows all such combinations along with their adjacency matrices. In the case of impact combinations of $n=1$, it is also difficult to talk about impact connectedness 

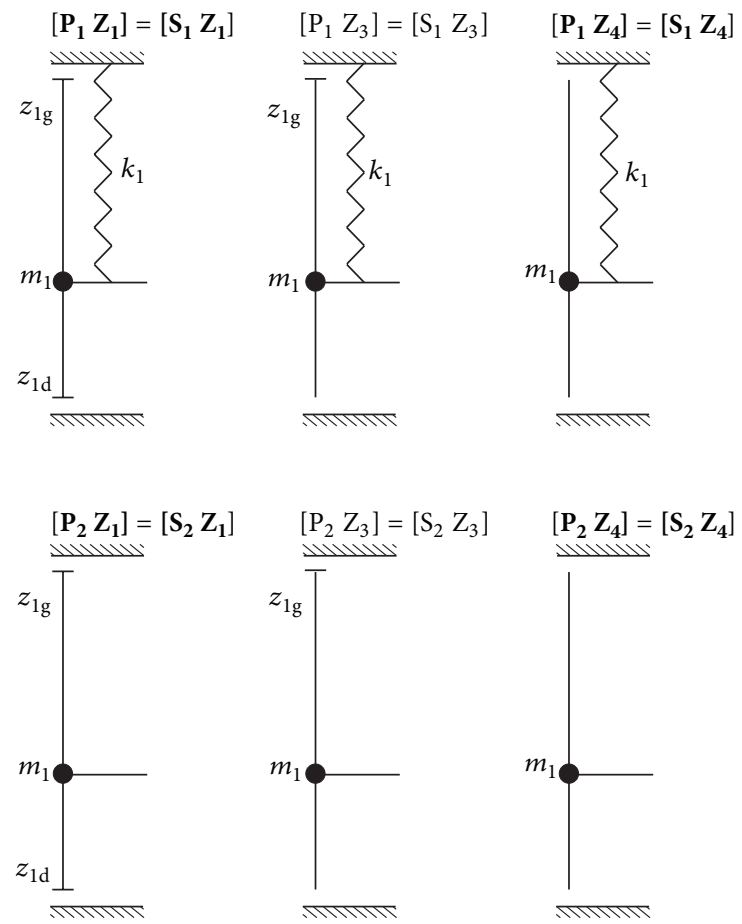

Figure 9: Connection-impact (spring-impact) combinations for $n=1$.

(subphase of Phase II): either the system does not have any fenders connecting it to the frame $\left(\mathbf{Z}_{4}\right.$ in Figure 8$)$, has one fender (lower $\mathbf{Z}_{2}$ in Figure 8 or upper $\mathbf{Z}_{3}$ in Figure 8 ) or has two such fenders $\left(\mathbf{Z}_{1}\right.$ in Figure 8$)$, but they do not belong to the impact connectedness zone. The following relations take place in them: the impact combination $\mathbf{Z}_{\mathbf{1}}$ is equivalent to itself, the $\mathbf{Z}_{\mathbf{4}}$ combination is equivalent to itself, and the $\mathbf{Z}_{2}$ combination is equivalent to the combination $\mathbf{Z}_{3}$ via transposition.

According to the assumed principle (Phase I), we match now each spring combination with each impact combination. As a result of the elimination of redundant equivalent combinations (via transposition), we obtain the spring-impact systems presented in Figure 9. As all spring and impact combinations with one degree-of-freedom are connected; thus all spring-impact systems formed as a result of matching such combinations are connected. In Figure 9 the systems in which there are no impact connections $\left(\mathbf{S}_{1} \mathbf{Z}_{\mathbf{4}}\right)$, spring connections $\left(\mathbf{S}_{\mathbf{2}} \mathbf{Z}_{\mathbf{1}}\right.$ and $\left.\mathrm{S}_{2} \mathrm{Z}_{3}\right)$, impact connections, and spring connections $\left(\mathbf{S}_{\mathbf{2}} \mathbf{Z}_{\mathbf{4}}\right)$ are marked.

Let us notice that systems with one degree-of-freedom can be treated as subsystems of disconnected systems with two degrees-of-freedom. The subsystems of matching spring systems would correspond to the systems shown in Figure 9: $\mathrm{S}_{7}$ (the spring connection of the system of a mass $m_{1}$ with the frame) and $\mathbf{S}_{\mathbf{8}}$ (there is no spring connection of the subsystem of a mass $m_{1}$ with the frame), with the following disconnected impact systems: $\mathbf{Z}_{55}$ (the impact connection via the fenders $z_{1 \mathrm{~g}}$ and $z_{1 d}$ of the subsystem of a mass $m_{1}$ with the frame), $\mathbf{Z}_{63}$ (the impact connection via the fender $z_{1 g}$ of the subsystem of a mass $m_{1}$ with the frame), and $\mathbf{Z}_{\mathbf{6 4}}$ (there is no impact connection of the subsystem of a mass $m_{1}$ with the frame).

\section{Examples of Systems Considered in the Literature and Their Structural Patterns}

Let is notice that instead of a spring connection, we can introduce any other connection that describes the action of at least one force (linear or nonlinear) that depends on displacement or velocity in the system. It cannot only be an elasticity force, but also a viscous damping force, a friction force, or an elastic-damping force or even a triple combination of these forces. As the occurrence of a fender does not determine the way the impact is modeled, thus the approach described here enables identification of all structural patterns of connection-impact systems for the given $n$. Let us draw attention to the fact that their number does not grow with an appearance of subsequent qualitatively different forces (dependent on displacement or velocity) or with a change in the impact modeling method. All the considerations conducted for spring-impact systems can be thus repeated without any obstacles for connection-impact systems. The obtained adjacency matrices of PZ connectionimpact systems are identical to the adjacency matrices of SZ spring-impact systems. Hence, we can state that for $n=$ $1\left(\mathrm{P}_{1} \mathrm{Z}_{1}, \mathrm{P}_{1} \mathrm{Z}_{3}, \mathrm{P}_{2} \mathrm{Z}_{1}\right.$, and $\mathrm{P}_{2} \mathrm{Z}_{3}$ in Figure 9) and $n=2$ (all connected representatives of classes of relations without $\mathrm{P}_{1} \mathrm{Z}_{64}, \mathrm{P}_{5} \mathrm{Z}_{64}$, and $\mathrm{P}_{6} \mathrm{Z}_{64}$; see Table 5); we have identified all structural patterns of connection-impact systems. Tables 6 and 7 include selected examples of mechanical one- and twodegrees-of-freedom systems chosen from the literature. for all systems, their structural patterns $S_{p}$ have been assigned according to the classification method proposed in [111]. Similarly, structural patterns for other examples from the literature can be defined. 
TABLE 7: Examples from the references $(n=2)$ and the notations of the structural pattern $\left(S_{p}\right)$ of the systems considered in them.

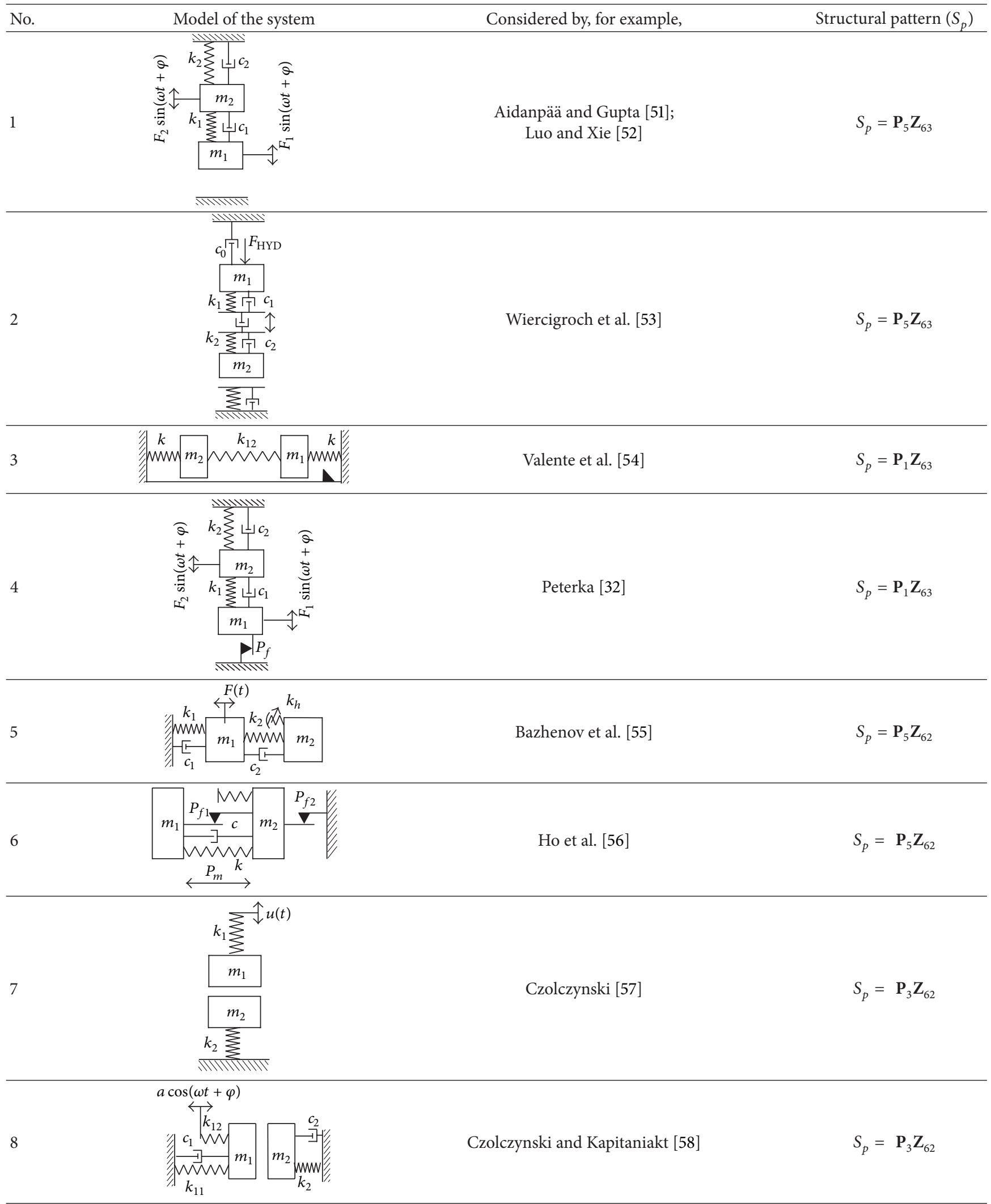


TABle 7: Continued.

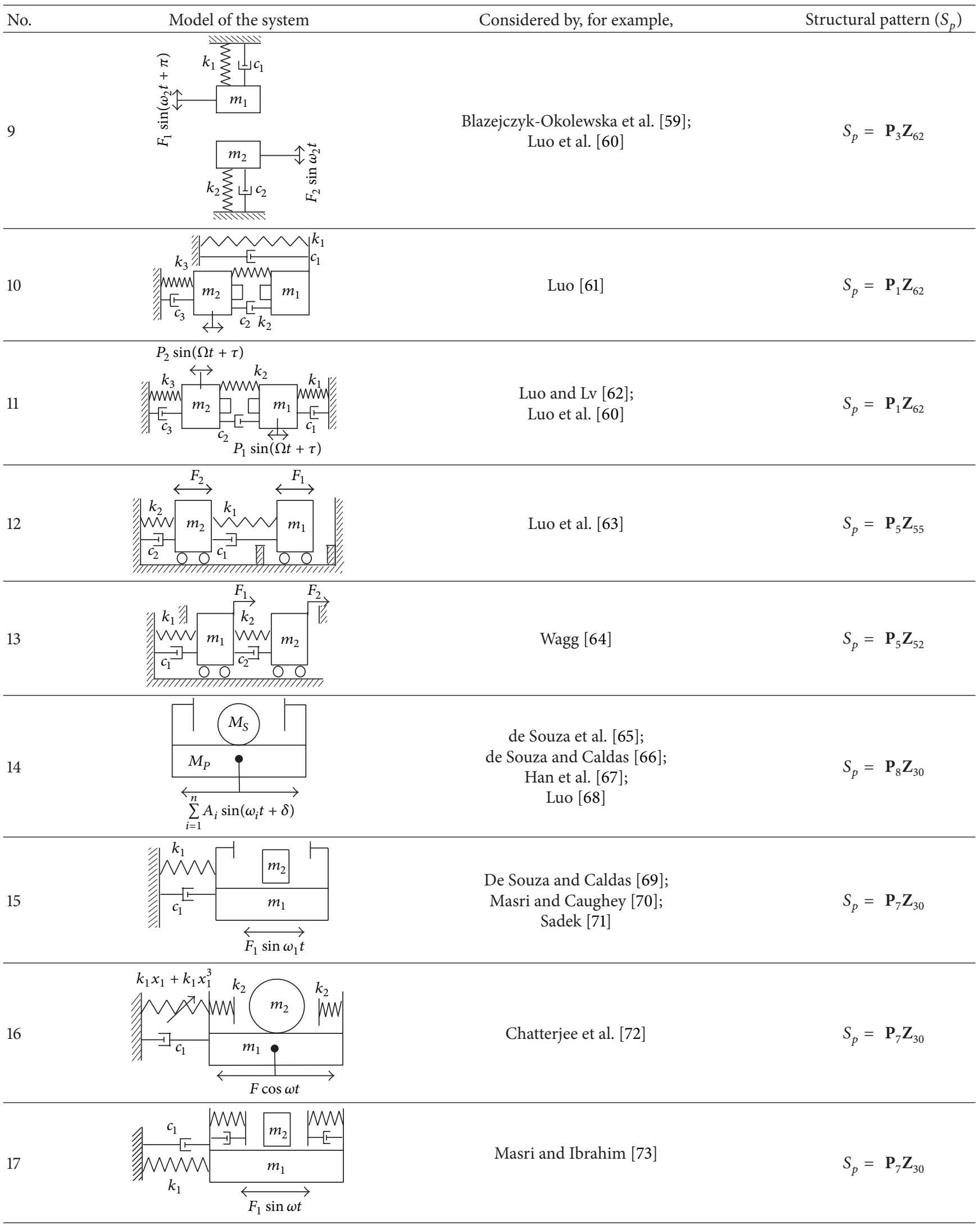


TABLE 7: Continued.

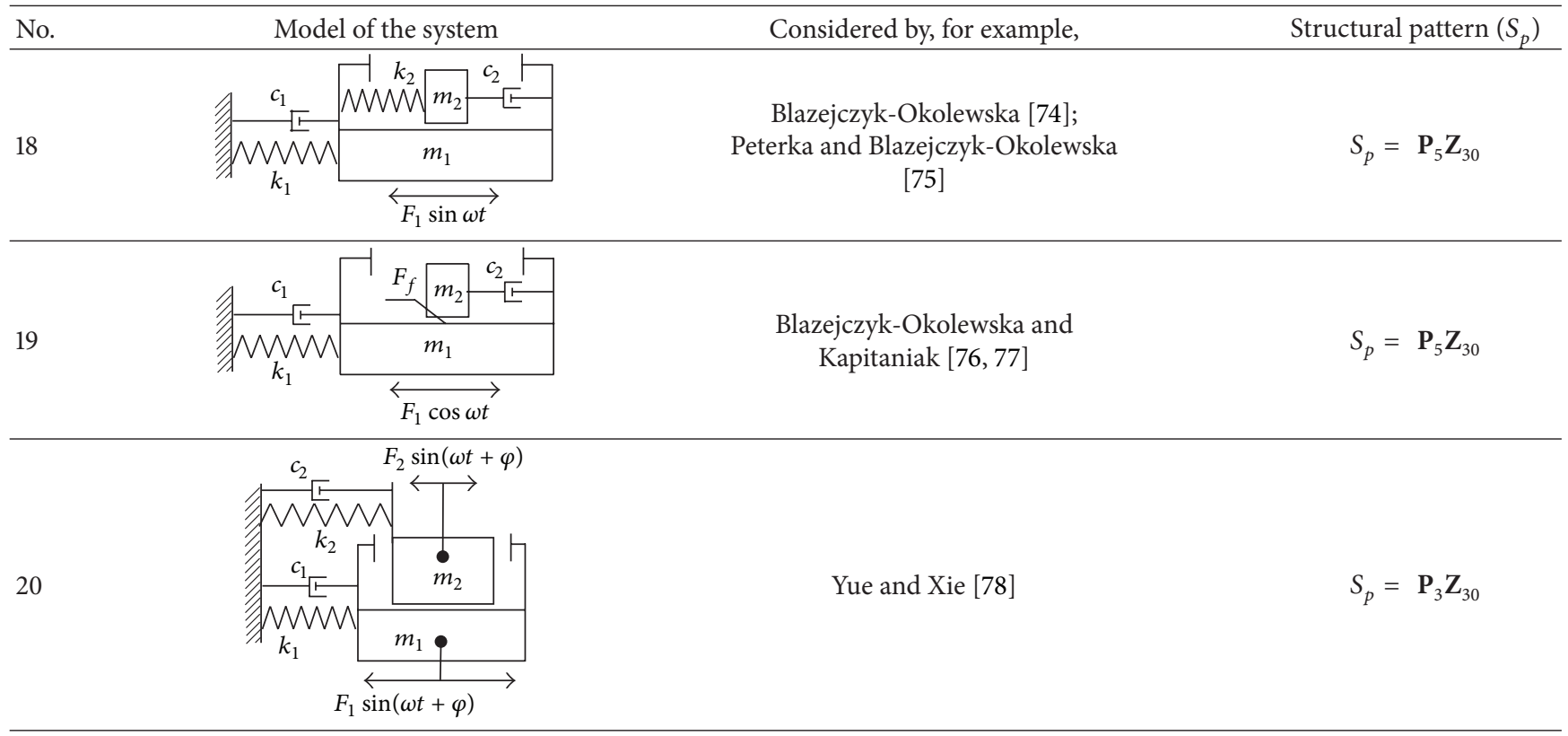

An introduction of arbitrary connections instead of spring connections also leads to the determination of all structural patterns for mechanical systems without impacts (referred to as oscillators) of $n=1\left(\mathrm{P}_{1} \mathrm{Z}_{4}\right.$ in Figure 9) and $n=2\left(\mathrm{P}_{1} \mathrm{Z}_{64}, \mathrm{P}_{5} \mathrm{Z}_{64}\right.$, and $\mathrm{P}_{6} \mathrm{Z}_{64}$ in Table 5). Such systems are commonly analyzed. As an example, we can take the $\mathrm{P}_{5} \mathrm{Z}_{64}$ pattern, which is considered to be a dynamic vibration damper in the majority of books on theory of vibrations of machines and devices (e.g., Den Hartog [112], Timoshenko [113]). On the other hand, with the $\mathrm{P}_{1} \mathrm{Z}_{4}$ (Figure 9) pattern, all systems with one degree-of-freedom can be described. The analysis of various oscillators of $n=1$ (linear and nonlinear) can be found, for example, in monographs by Den Hartog [112], Timoshenko [113], Kapitaniak [114], and Guckenheimer and Holmes [101].

\section{Conclusions}

In the study, the classification method developed by Blazejczyk-Okolewska [111] has been illustrated in the example of mechanical systems with one and two degreesof-freedom. The relations occurring in the system have been described in terms of the matrix representation. The identification and the elimination of redundant adjacency matrices and the identification of connected systems have been conducted with the previously developed procedures. According to the principles of selection, the connected representatives of connection-impact classes of relations have been determined. They constitute a set of all structural patterns of vibroimpact systems. Four patterns of impact oscillators with one degree-of-freedom and 141 patterns of impact oscillators with two degrees-of-freedom have been distinguished. The knowledge of classification principles enables classification of each mechanical system with impacts with arbitrary connections. The procedure for classification of the given system has been presented in numerous examples taken from the literature devoted to the subject.

The considerations conducted for systems with impacts have allowed for defining the number of patterns of systems without impacts, commonly referred to as oscillators. In this case, one pattern with one degree-of-freedom and three patterns with two degrees-of-freedom have been distinguished.

The proposed classification of mechanical systems with impacts according to characteristic properties of their structure seems to be a natural classification. It reflects the relationships between the system structures, tells us about their way of evolution, and presents their genesis. It allows us to rearrange the knowledge on systems with impacts and is the basis for understanding the sources of their diversity. Providing a full set of objects to be analyzed, it gives hints for new ideas and directions in designing technical devices.

\section{References}

[1] U. Andreaus, L. Placidi, and G. Rega, "Numerical simulation of the soft contact dynamics of an impacting bilinear oscillator," Communications in Nonlinear Science and Numerical Simulation, vol. 15, no. 9, pp. 2603-2616, 2010.

[2] J. Awrejcewicz, K. Tomczak, and C. H. Lamarque, "Controlling systems with impacts," International Journal of Bifurcation and Chaos, vol. 9, no. 3, pp. 547-553, 1999.

[3] A. Bichri, M. Belhaq, and J. Perret-Liaudet, "Control of vibroimpact dynamics of a single-sided Hertzian contact forced oscillator," Nonlinear Dynamics, vol. 63, no. 1-2, pp. 51-60, 2011.

[4] G. W. Blankenship and A. Kahraman, "Steady state forced response of a mechanical oscillator with combined parametric excitation and clearance type non-linearity," Journal of Sound and Vibration, vol. 185, no. 5, pp. 743-765, 1995. 
[5] C. Cempel, The Periodical Vibration with Impacts in Mechanical Discreet Systems, Dissertation Series no. 44, Technical University of Poznan, Poznan, Poland, 1970.

[6] W. Chin, E. Ott, H. E. Nusse, and C. Grebogi, "Grazing bifurcations in impact oscillators," Physical Review E, vol. 50, no. 6, pp. 4427-4444, 1994.

[7] W. Chin, E. Ott, H. E. Nusse, and C. Grebogi, "Universal behavior of impact oscillators near grazing incidence," Physics Letters A, vol. 201, no. 2-3, pp. 197-204, 1995.

[8] J. de Weger, W. van de Water, and J. Molenaar, "Grazing impact oscillations," Physical Review E, vol. 62, no. 2, pp. 2030-2041, 2000.

[9] M. Di Bernardo, M. I. Feigin, S. J. Hogan, and M. E. Homer, "Local analysis of C-bifurcations in n-dimensional piecewisesmooth dynamical systems," Chaos, Solitons \& Fractals, vol. 10, no. 11, pp. 1881-1908, 1999.

[10] W. Fang and J. A. Wickert, "Response of a periodically driven impact oscillator," Journal of Sound and Vibration, vol. 170, no. 3, pp. 397-409, 1994.

[11] Y. S. Fedosenko and M. I. Feigin, "Periodic motion of vibrating hammer including the presence of sliding regime," Prikladnaja Matematika i Mekhanika, vol. 35, no. 5, pp. 892-898, 1971 (Russian).

[12] M. I. Feigin, "Period-doubling at C-bifurcation in the piecewise-continuous systems," Prikladnaja Matematika $i$ Mekhanika, vol. 34, pp. 861-869, 1970 (Russian).

[13] S. Foale and S. R. Bishop, "Bifurcations in impact oscillations," Nonlinear Dynamics, vol. 6, no. 3, pp. 285-299, 1994.

[14] N. Hinrichs, M. Oestreich, and K. Popp, "Dynamics of oscillators with impact and friction," Chaos, Solitons \& Fractals, vol. 8, no. 4, pp. 535-558, 1997.

[15] K. H. Hunt and F. R. E. Crossley, "Coefficient of restitution interpreted as damping in vibroimpact," Journal of Applied Mechanics, vol. 42, no. 2, pp. 440-445, 1975.

[16] H. M. Isomäki, J. Von Boehm, and R. Räty, "Devil's attractors and chaos of a driven impact oscillator," Physics Letters A, vol. 107, no. 8, pp. 343-346, 1985.

[17] A. P. Ivanov, "Stabilization of an impact oscillator near grazing incidence owing to resonance," Journal of Sound and Vibration, vol. 162, no. 3, pp. 562-565, 1993.

[18] A. P. Ivanov, "Impact oscillations: linear theory of stability and bifurcations," Journal of Sound and Vibration, vol. 178, no. 3, pp. 361-378, 1994.

[19] A. Kahraman and R. Singh, "Dynamics of an oscillator with both clearance and continuous non-linearities," Journal of Sound and Vibration, vol. 153, no. 1, pp. 180-185, 1992.

[20] A. E. Kobrinskii and A. Kobrinskii, Vibroimpact Systems, Nauka Press, Moscow, Russia, 1973.

[21] M. Z. Kolovskij, Nonlinear Theory of Impact Systems, Nauka Press, Moscow, Russia, 1966.

[22] S. Kundu, S. Banerjee, J. Ing, E. Pavlovskaia, and M. Wiercigroch, "Singularities in soft-impacting systems," Physica D, vol. 231, no. 5, pp. 553-565, 2011.

[23] J. Y. Lee and J. J. Yan, "Position control of double-side impact oscillator," Mechanical Systems and Signal Processing, vol. 21, no. 2, pp. 1076-1083, 2007.

[24] R. I. Leine and D. H. van Campen, "Discontinuous bifurcations of periodic solutions," Mathematical and Computer Modelling, vol. 36, no. 3, pp. 259-273, 2002.
[25] Q. H. Li and Q. S. Lu, "Coexisting periodic orbits in vibro-impacting dynamical systems," Applied Mathematics and Mechanics, vol. 24, no. 3, pp. 261-273, 2003.

[26] S. Q. Lin and C. N. Bapat, "Estimation of clearances and impact forces using vibroimpact response: random excitation," Journal of Sound and Vibration, vol. 163, no. 3, pp. 407-421, 1993.

[27] A. C. J. Luo and S. Menon, "Global chaos in a periodically forced, linear system with a dead-zone restoring force," Chaos, Solitons \& Fractals, vol. 19, no. 5, pp. 1189-1199, 2004.

[28] F. C. Moon and S. W. Shaw, "Chaotic vibrations of a beam with non-linear boundary conditions," International Journal of NonLinear Mechanics, vol. 18, no. 6, pp. 465-477, 1983.

[29] A. B. Nordmark, "Non-periodic motion caused by grazing incidence in an impact oscillator," Journal of Sound and Vibration, vol. 145, no. 2, pp. 279-297, 1991.

[30] A. B. Nordmark, "Effects due to low velocity impact in mechanical oscillators," International Journal of Bifurcation and Chaos, vol. 2, no. 3, pp. 597-605, 1992.

[31] E. Pavlovskaia, M. Wiercigroch, and C. Grebogi, "Modeling of an impact system with a drift," Physical Review E, vol. 64, no. 5, Article ID 056224, 9 pages, 2001.

[32] F. Peterka, "An investigation of the motion of impact dampers, paper I, II, III," StrojniCku Casopis, vol. 21, no. 5, 1971 (Czech).

[33] F. Peterka, Introduction to Vibration of Mechanical Systems with Internal Impacts, Academia, Praha, Czech Republic, 1981.

[34] F. Peterka and J. Vacík, "Transition to chaotic motion in mechanical systems with impacts," Journal of Sound and Vibration, vol. 154, no. 1, pp. 95-115, 1992.

[35] L. Půst and F. Peterka, "Impact oscillator with Hertz's model of contact," Meccanica, vol. 38, no. 1, pp. 99-114, 2003.

[36] S. W. Shaw, "The dynamics of a harmonically excited systems having rigid amplitude constraints-part I, part II," Journal of Applied Mechanics, vol. 52, no. 2, pp. 459-464, 1985.

[37] S. W. Shaw and P. J. Holmes, "A periodically forced piecewise linear oscillator," Journal of Sound and Vibration, vol. 90, no. 1, pp. 129-155, 1983.

[38] S. W. Shaw and P. J. Holmes, "A periodically forced impact oscillator with large dissipation," Journal of Applied Mechanics, vol. 50, no. 4, pp. 849-857, 1983.

[39] S. W. Shaw and P. Holmes, "Periodically forced linear oscillator with impacts: chaos and long-period motions," Physical Review Letters, vol. 51, no. 8, pp. 623-626, 1983.

[40] A. Stefanski and T. Kapitaniak, "Using chaos synchronization to estimate the largest Lyapunov exponent of nonsmooth systems," Discrete Dynamics in Nature and Society, vol. 4, no. 3, pp. 207215, 2000.

[41] A. Stefanski and T. Kapitaniak, "Estimation of the dominant Lyapunov exponent of non-smooth systems on the basis of maps synchronization," Chaos, Solitons \& Fractals, vol. 15, no. 2, pp. 233-244, 2003.

[42] J. M. T. Thompson and R. Ghaffari, "Chaos after perioddoubling bifurcations in the resonance of an impact oscillator," Physics Letters A, vol. 91, no. 1, pp. 5-8, 1982.

[43] P. C. Tung, "The dynamics of a nonharmonically excited system having rigid amplitude constraints," Journal of Applied Mechanics, vol. 59, no. 3, pp. 693-695, 1992.

[44] G. S. Whiston, "Global dynamics of a vibro-impacting linear oscillator," Journal of Sound and Vibration, vol. 118, no. 3, pp. 395-424, 1987.

[45] A. M. Krivtsov and M. Wiercigroch, "Dry friction model of percussive drilling," Meccanica, vol. 34, no. 6, pp. 425-435, 1999. 
[46] O. K. Ajibose, M. Wiercigroch, E. Pavlovskaia, and A. R. Akisanya, "Global and local dynamics of drifting oscillator for different contact force models," International Journal of NonLinear Mechanics, vol. 45, no. 9, pp. 850-858, 2010.

[47] V. I. Babickiü, Theory of Vibroimpact Systems, Nauka, Moscow, Russia, 1978.

[48] Y. Ma, J. Ing, S. Banerjee, M. Wiercigroch, and E. Pavlovskaia, "The nature of the normal form map for soft impacting systems," International Journal of Non-Linear Mechanics, vol. 43, no. 6, pp. 504-513, 2008.

[49] J. Ing, E. Pavlovskaia, M. Wiercigroch, and S. Banerjee, "Bifurcation analysis of an impact oscillator with a one-sided elastic constraint near grazing," Physica D, vol. 239, no. 6, pp. 312-321, 2010.

[50] M. Wiercigroch and V. W. T. Sin, "Experimental study of a symmetrical piecewise base-excited oscillator," Journal of Applied Mechanics, vol. 65, no. 3, pp. 657-663, 1998.

[51] J. O. Aidanpää and R. B. Gupta, "Periodic and chaotic behaviour of a threshold-limited two-degree-of-freedom system," Journal of Sound and Vibration, vol. 165, no. 2, pp. 305-327, 1993.

[52] G. W. Luo and J. H. Xie, "Hopf bifurcations and chaos of a two-degree-of-freedom vibro-impact system in two strong resonance cases," International Journal of Non-Linear Mechanics, vol. 37, no. 1, pp. 19-34, 2002.

[53] M. Wiercigroch, R. D. Neilson, and M. A. Player, "Material removal rate prediction for ultrasonic drilling of hard materials using an impact oscillator approach," Physics Letters A, vol. 259, no. 2, pp. 91-96, 1999.

[54] A. X. C. N. Valente, N. H. McClamroch, and I. Mezić, "Hybrid dynamics of two coupled oscillators that can impact a fixed stop," International Journal of Non-Linear Mechanics, vol. 38, no. 5, pp. 677-689, 2003.

[55] V. A. Bazhenov, O. S. Pogorelova, T. G. Postnikova, and O. A. Luk'yanchenko, "Numerical investigations of the dynamic processes in vibroimpact systems in modeling impacts by a force of contact interaction," Strength of Materials, vol. 40, no. 6, pp. 656-662, 2008.

[56] J. H. Ho, V. D. Nguyen, and K. C. Woo, "Nonlinear dynamics of a new electro-vibro-impact system," Nonlinear Dynamics, vol. 63, no. 1-2, pp. 35-49, 2011.

[57] K. Czolczynski, "On the existence of a stable periodic motion of two impacting oscillators," Chaos, Solitons \& Fractals, vol. 15, no. 2, pp. 371-379, 2003.

[58] K. Czolczynski and T. Kapitaniakt, "On the existence of a stable periodic solution of two impacting oscillators with damping," International Journal of Bifurcation and Chaos, vol. 14, no. 11, pp. 3931-3947, 2004.

[59] B. Blazejczyk-Okolewska, J. Brindley, K. Czolczynski, and T. Kapitaniak, "Antiphase synchronization of chaos by noncontinuous coupling: two impacting oscillators," Chaos, Solitons \& Fractals, vol. 12, no. 10, pp. 1823-1826, 2001.

[60] G. W. Luo, Y. L. Zhang, and J. N. Yu, "Dynamical behavior of vibro-impact machinery near a point of codimension two bifurcation," Journal of Sound and Vibration, vol. 292, no. 1-2, pp. 242-278, 2006.

[61] G. W. Luo, "Dynamics of an impact-forming machine," International Journal of Mechanical Sciences, vol. 48, no. 11, pp. 12951313, 2006.

[62] G. W. Luo and X. H. Lv, "Controlling bifurcation and chaos of a plastic impact oscillator," Nonlinear Analysis: Real World Applications, vol. 10, no. 4, pp. 2047-2061, 2009.
[63] G. Luo, L. Ma, and X. Lv, "Dynamic analysis and suppressing chaotic impacts of a two-degree-of-freedom oscillator with a clearance," Nonlinear Analysis: Real World Applications, vol. 10, no. 2, pp. 756-778, 2009.

[64] D. J. Wagg, "Periodic sticking motion in a two-degree-offreedom impact oscillator," International Journal of Non-Linear Mechanics, vol. 40, no. 8, pp. 1076-1087, 2005.

[65] S. L. T. de Souza, A. M. Batista, I. L. Caldas, R. L. Viana, and T. Kapitaniak, "Noise-induced basin hopping in a vibro-impact system," Chaos, Solitons \& Fractals, vol. 32, no. 2, pp. 758-767, 2007.

[66] S. L. T. de Souza and I. L. Caldas, "Controlling chaotic orbits in mechanical systems with impacts," Chaos, Solitons \& Fractals, vol. 19, no. 1, pp. 171-178, 2004.

[67] R. P. S. Han, A. C. J. Luo, and W. Deng, "Chaotic motion of a horizontal impact pair," Journal of Sound and Vibration, vol. 181, no. 2, pp. 231-250, 1995.

[68] A. C. J. Luo, "Period-doubling induced chaotic motion in the LR model of a horizontal impact oscillator," Chaos, Solitons \& Fractals, vol. 19, no. 4, pp. 823-839, 2004.

[69] S. L. T. de Souza and I. L. Caldas, "Calculation of Lyapunov exponents in systems with impacts," Chaos, Solitons \& Fractals, vol. 19, no. 3, pp. 569-579, 2004.

[70] S. F. Masri and T. K. Caughey, "On the stability of the impact damper," Journal of Applied Mechanics, vol. 33, pp. 586-592, 1966.

[71] M. M. Sadek, "The behaviour of the impact damper," Proceedings of the Institution of Mechanical Engineers, vol. 180, pp. 895906, 1965.

[72] S. Chatterjee, A. K. Mallik, and A. Ghosh, "On impact dampers for non-linear vibrating sytsems," Journal of Sound and Vibration, vol. 187, no. 3, pp. 403-420, 1995.

[73] S. F. Masri and A. M. Ibrahim, "Stochastic excitation of a simple system with impact damper," Earthquake Engineering and Structural Dynamics, vol. 1, pp. 337-346, 1973.

[74] B. Blazejczyk-Okolewska, "Analysis of an impact damper of vibrations," Chaos, Solitons \& Fractals, vol. 12, no. 11, pp. 19831988, 2001.

[75] F. Peterka and B. Blazejczyk-Okolewska, "Some aspects of the dynamical behavior of the impact damper," Journal of Vibration and Control, vol. 11, no. 4, pp. 459-479, 2005.

[76] B. Blazejczyk-Okolewska and T. Kapitaniak, "Dynamics of impact oscillator with dry friction," Chaos, Solitons \& Fractals, vol. 7, no. 9, pp. 1455-1459, 1996.

[77] B. Blazejczyk-Okolewska and T. Kapitaniak, "Co-existing attractors of impact oscillator," Chaos, Solitons \& Fractals, vol. 9, no. 8, pp. 1439-1443, 1998.

[78] Y. Yue and J. H. Xie, "Symmetry and bifurcations of a twodegree-of-freedom vibro-impact system," Journal of Sound and Vibration, vol. 314, no. 1-2, pp. 228-245, 2008.

[79] W. Goldsmith, Impact: The Theory and Physical Behaviour of Colliding Solids, Edward Arnold Publishers Ltd., London, UK, 1960.

[80] Y. B. Kim and S. T. Noah, "Stability and bifurcation analysis of oscillators with piecewise-linear characteristics. A general approach," Journal of Applied Mechanics, vol. 58, no. 2, pp. 545553, 1991.

[81] S. L. Lau and W. S. Zhang, "Nonlinear vibrations of piecewiselinear systems by incremental harmonic balance method," Journal of Applied Mechanics, vol. 59, no. 1, pp. 153-160, 1992. 
[82] S. Narayanan and P. Sekar, "Periodic and chaotic responses of an SDF system with piecewise linear stiffness subjected to combined harmonic and flow induced excitations," Journal of Sound and Vibration, vol. 184, no. 2, pp. 281-298, 1995.

[83] S. Natsiavas, "Dynamics of piecewise linear oscillators with van der Pol type damping," International Journal of Non-Linear Mechanics, vol. 26, no. 3-4, pp. 349-366, 1991.

[84] Y. Wang, "Dynamics of unsymmetric piecewise-linear/nonlinear systems using finite elements in time," Journal of Sound and Vibration, vol. 185, no. 1, pp. 155-170, 1995.

[85] C. W. Wong, W. S. Zhang, and S. L. Lau, "Periodic forced vibration of unsymmetrical piecewise-linear systems by incremental harmonic balance method," Journal of Sound and Vibration, vol. 149, no. 1, pp. 91-105, 1991.

[86] S. F. Masri, "Analytical and experimental studies of multiunit impact dampers," The Journal of the Acoustical Society of America, vol. 45, no. 5, pp. 1111-1117, 1964.

[87] Y. Araki, I. Yokomichi, and Y. Jinnouchi, "Impact damper with granular materials: 4th report frequency response in a horizontal system," Bulletin of the Japanese Society of Mechanical Engineers, vol. 29, no. 258, pp. 4334-4338, 1986.

[88] C. N. Bapat and S. Sankar, "Multiunit impact damper-reexamined," Journal of Sound and Vibration, vol. 103, no. 4, pp. 457-469, 1985.

[89] E. V. Karpenko, E. E. Pavlovskaia, and M. Wiercigroch, "Bifurcation analysis of a preloaded Jeffcott rotor," Chaos, Solitons \& Fractals, vol. 15, no. 2, pp. 407-416, 2003.

[90] E. V. Karpenko, M. Wiercigroch, E. E. Pavlovskaia, and M. P. Cartmell, "Piecewise approximate analytical solutions for a Jeffcott rotor with a snubber ring," International Journal of Mechanical Sciences, vol. 44, no. 3, pp. 475-488, 2002.

[91] E. V. Karpenko, M. Wiercigroch, E. E. Pavlovskaia, and R. D. Neilson, "Experimental verification of Jeffcott rotor model with preloaded snubber ring," Journal of Sound and Vibration, vol. 298, no. 4-5, pp. 907-917, 2006.

[92] K. Koizumi, Analysis of vibration with collision and applications to leaf beating machine [Doctor thesis], University of Tokyo, Precision Mechanical Engineering, 1980.

[93] W. H. Park, "Mass-spring-damper response to repetitive impacts," Journal of Engineering for Industry, vol. 89, no. 4, pp. 587-596, 1967.

[94] J. Jerrelind and A. Stensson, "Nonlinear dynamics of parts in engineering systems," Chaos, Solitons \& Fractals, vol. 11, no. 13, pp. 2413-2428, 2000.

[95] R. M. Brach, Mechanical Impact Dynamics: Rigid Body Collisions, John Wiley and Sons, 1991.

[96] G. Chen, Controlling Chaos and Bifurcations in Engineering Systems, CRC Press, Boca Raton, Fla, USA, 2000.

[97] J. Awrejcewicz and C.-H. Lamarque, Bifurcation and Chaos in Nonsmooth Mechanical Systems, vol. 45 of World Scientific Series on Nonlinear Science. Series A: Monographs and Treatises, World Scientific Publishing, River Edge, NJ, USA, 2003.

[98] S. R. Bishop, "Impact oscillators," Philosophical Transactions of the Royal Society A, vol. 347, no. 1683, pp. 347-351, 1994.

[99] B. Blazejczyk-Okolewska, K. Czolczynski, T. Kapitaniak, and J. Wojewoda, Chaotic Mechanics in Systems with Impacts and Friction, vol. 36 of World Scientific Series on Nonlinear Science. Series A: Monographs and Treatises, World Scientific Publishing, Singapore, 1999.

[100] B. Brogliato, Nonsmooth Mechanics, Springer, 1999.
[101] J. Guckenheimer and P. Holmes, Nonlinear Oscillations, Dynamical Systems, and Bifurcations of Vector Fields, vol. 42 of Applied Mathematical Sciences, Springer, New York, NY, USA, 1983.

[102] R. A. Ibrahim, Vibro-Impact Dynamics: Modeling, Mapping and Applications, vol. 43 of Lecture Notes in Applied and Computational Mechanics, Springer, Berlin, Germany, 2009.

[103] G. X. Li, R. H. Rand, and F. C. Moon, "Bifurcations and chaos in a forced zero-stiffness impact oscillator," International Journal of Non-Linear Mechanics, vol. 25, no. 4, pp. 417-432, 1990.

[104] J. H. T. Thompson and S. R. Bishop, Nonlinearity and Chaos in Engineering Dynamics, John Wiley and Sons, 1994.

[105] E. Gutiérrez and D. K. Arrowsmith, "Control of a double impacting mechanical oscillator using displacement feedback," International Journal of Bifurcation and Chaos, vol. 14, no. 9, pp. 3095-3113, 2004.

[106] S. L. T. de Souza, M. Wiercigroch, I. L. Caldas, and J. M. Balthazar, "Suppressing grazing chaos in impacting system by structural nonlinearity," Chaos, Solitons \& Fractals, vol. 38, no. 3, pp. 864-869, 2008.

[107] A. C. J. Luo, "On flow switching bifurcations in discontinuous dynamical systems," Communications in Nonlinear Science and Numerical Simulation, vol. 12, no. 1, pp. 100-116, 2007.

[108] W. L. Zhao and L. Z. Wang, Random Fatigue and Clearance Nonlinearity of Mechanical Vibrating Systems, Science Publishing House, Beijing, China, 2007.

[109] P. B. Zinjade and A. K. Mallik, "Impact damper for controlling friction-driven oscillations," Journal of Sound and Vibration, vol. 306, no. 1-2, pp. 238-251, 2007.

[110] B. Blazejczyk-Okolewska, K. Czolczynski, and T. Kapitaniak, "Classification principles of types of mechanical systems with impacts-fundamental assumptions and rules," European Journal of Mechanics A/Solids, vol. 23, no. 3, pp. 517-537, 2004.

[111] B. Blazejczyk-Okolewska, "A method to determine structural patterns of mechanical systems with impacts," http://arxiv.org/ abs/1212.0193.

[112] J. P. Den Hartog, Mechanical Vibration, McGraw-Hill Book Company, 1956.

[113] S. Timoshenko, Vibration Problems in Engineering, D. Van Nostrand Company, Inc., 1956.

[114] T. Kapitaniak, Chaotic Oscillations in Mechanical Systems, Nonlinear Science: Theory and Applications, Manchester University Press, Manchester, UK, 1991. 


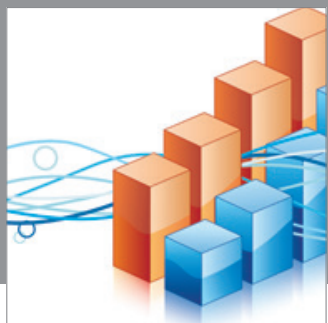

Advances in

Operations Research

mansans

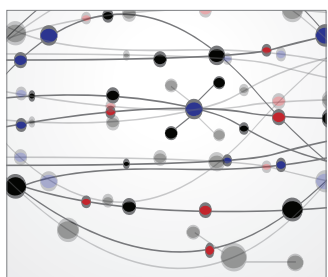

The Scientific World Journal
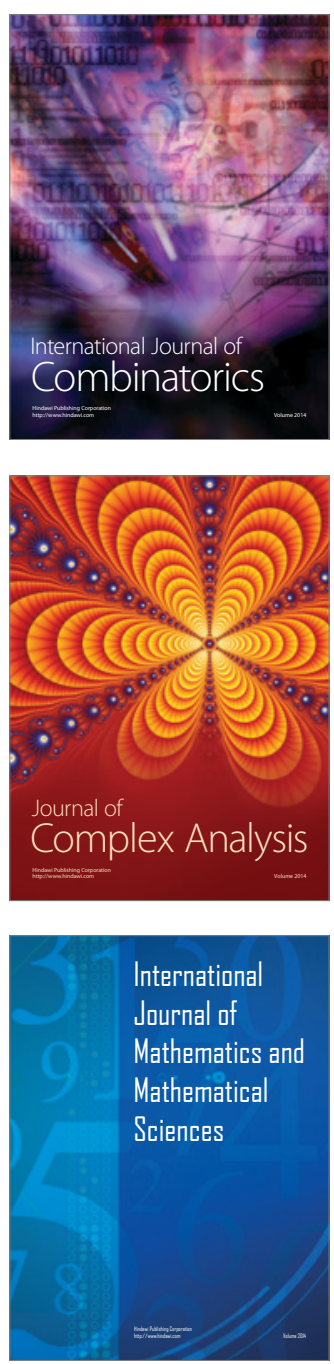
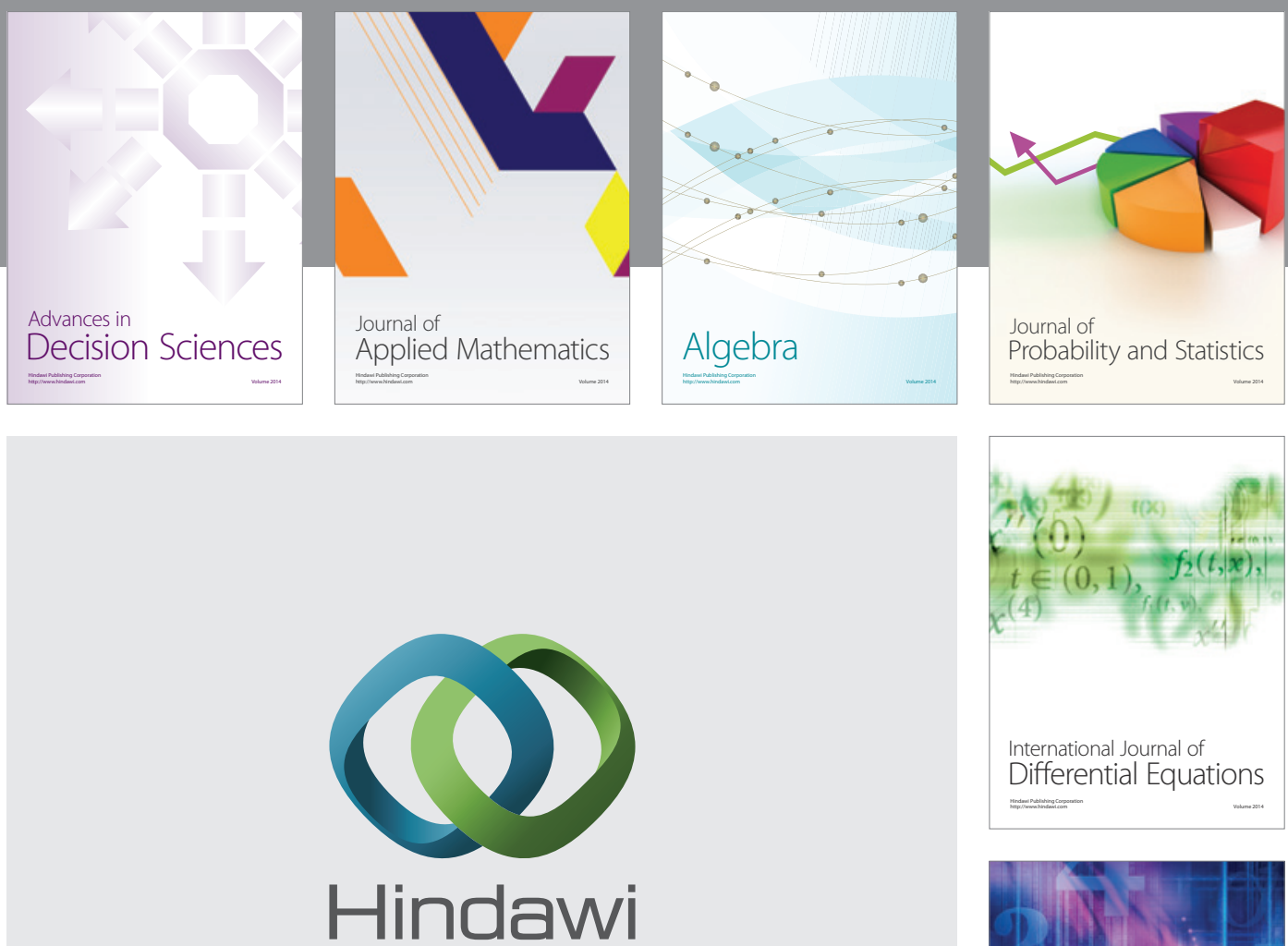

Submit your manuscripts at http://www.hindawi.com
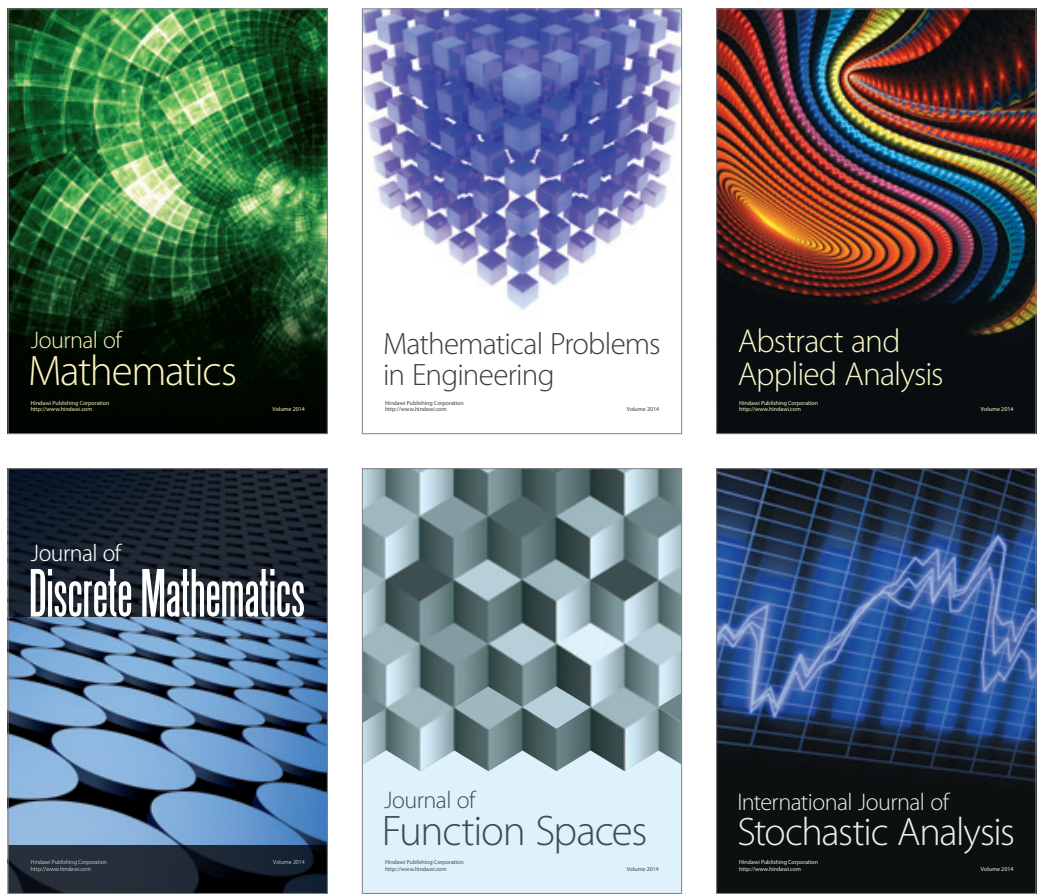

Journal of

Function Spaces

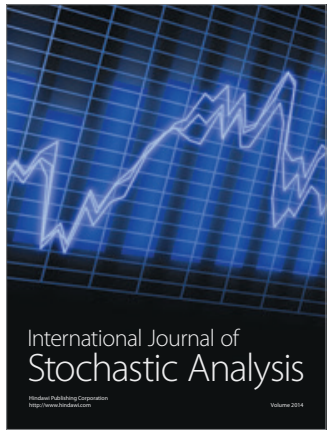

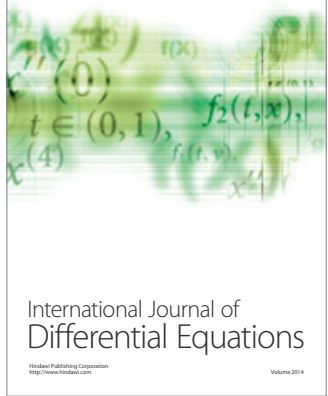
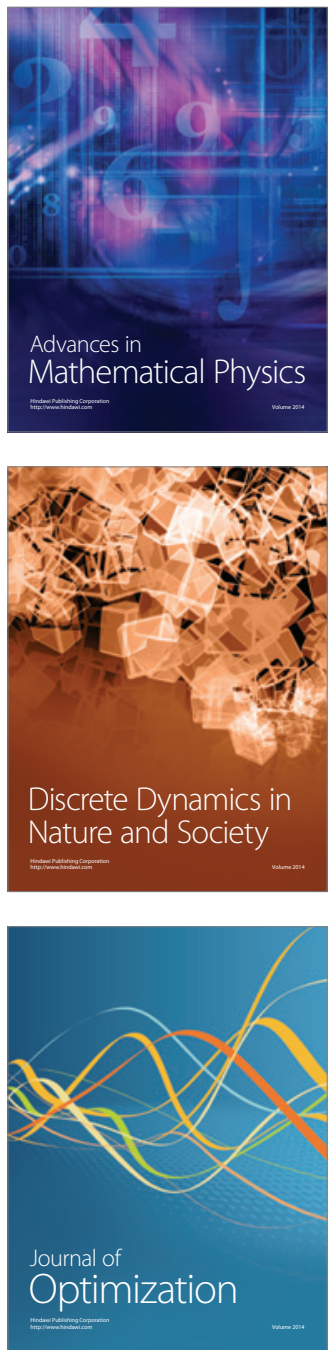